\title{
Large amplitude oscillatory microrheology
}

James W. Swan, Roseanna N. Zia, and John F. Brady

Citation: Journal of Rheology (1978-present) 58, 1 (2014); doi: 10.1122/1.4826939

View online: http://dx.doi.org/10.1122/1.4826939

View Table of Contents: http://scitation.aip.org/content/sor/journal/jor2/58/1?ver=pdfcov

Published by the The Society of Rheology

\section{Re-register for Table of Content Alerts}

Create a profile. 


\title{
Large amplitude oscillatory microrheology
}

\author{
James W. Swan ${ }^{a), b)}$ \\ Department of Chemical and Biomolecular Engineering, University of Delaware, \\ Newark, Delaware 19716 and Division of Chemistry and Chemical Engineering, \\ California Institute of Technology, Pasadena, California 91125
}

Roseanna N. Zia

School of Chemical and Biomolecular Engineering, Cornell University, Ithaca, New York 14850

John F. Brady

Division of Chemistry and Chemical Engineering, California Institute of Technology, Pasadena, California 91125 and Division of Engineering and Applied Science, California Institute of Technology, Pasadena, California 91125

(Received 13 August 2013; final revision received 10 October 2013; published 5 November 2013)

\begin{abstract}
Synopsis
We study the motion of a colloidal particle as it is driven by an oscillating external force of arbitrary amplitude and frequency through a colloidal dispersion. Large amplitude oscillatory flows (LAOFs) are examined predominantly from a phenomenological perspective in which experimental measurements inform constitutive models. Here, we investigate a LAOF from a microstructural perspective by connecting motion of the probe particle to the material response while making no assumptions a priori about how stress relaxes in the material. The suspension exerts nonconservative, hydrodynamic forces on the probe, while distortions in the particle configuration exert conservative forces: Brownian and interparticle forces, for example. The relative importance of each of these contributions to particle motion evolves with the degree of displacement from equilibrium. When the force on the probe is weak, the linear microviscoelasticity of the suspension is probed [see, e.g., Khair and Brady, J. Rheol. 49, 1449-1481 (2005)]. When oscillation rate is slow, the steady microrheology is probed [see, e.g., Squires and Brady, Phys. Fluids 17, 073101 (2005); Khair and Brady, J. Fluid Mech. 557, 73-117 (2006)]. This article develops a micromechanical model that recovers these limiting cases and then uses the same model to reveal the microrheology of colloidal dispersions deformed by a probe driven with arbitrary force amplitude and frequency. A chief result of this work is the discovery of a regime in which the resistance to motion of the probe particle is on average weaker than the resistance the probe experiences when deformed by high frequency oscillation. This hypoviscous effect arises when the reciprocating motion of the probe particle opens a
\end{abstract}

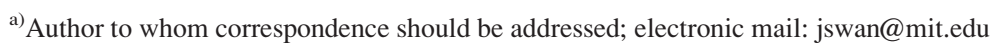

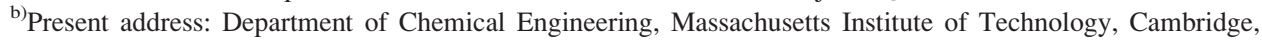
Massachusetts 02139.
} 
channel free of other particles which is thus less resistive to probe motion. This effect is most apparent under the conditions of strong forces, rapid oscillation, and large extent of deformation. (C) 2014 The Society of Rheology. [http://dx.doi.org/10.1122/1.4826939]

\section{INTRODUCTION}

Complex fluids play an important role in many aspects of daily life, from foodstuffs to pharmaceutical materials, and from toothpaste to the mucus that allows our lungs to reinflate at the end of a breath. As a class of materials, complex fluids span a very broad range of soft matter but share a common fundamental structure: A collection of microscopic particles or domains - a microstructure-embedded in a macroscopic domain, which relaxes over familiar and observable time scales. The presence and configuration of this internal microstructure imparts to the material a rich spectrum of equilibrium and nonequilibrium mechanical properties. Imposing a flow on such a material distorts the equilibrium microstructure, which can give rise to profound changes in flow behaviorincluding dramatic increases or decreases in viscosity and time-dependent behaviors such as viscoelasticity. In traditional macroscale rheology, bulk material displacements are induced via shearing or extensional flows. A constitutive relation between the rate of deformation and the stress may then be obtained. In theoretical approaches, the study of steady-state rheology over a range of flow strengths connects macroscopic nonNewtonian behavior with its underlying microstructural origins. For example, in colloidal dispersions an increase in flow strength is accompanied by a decrease in the relative importance of Brownian diffusion. For weak flows, this gives rise to shear thinning [Batchelor (1976); Bergenholtz et al. (2002)]. As flow strength continues to increase, hydrodynamic interactions dominate the microstructural deformation, resulting in the onset of shear thickening [Brady and Bossis (1985); Barnes (1989); D'Haene et al. (1993); Bender and Wagner (1995); Maranzano and Wagner (2002); Bergenholtz et al. (2002); Wagner and Brady (2009)]. Recent experimental studies report direct observations of particle microstructure in real (rather than wave) space as well, along with computation of the stress via a statistical mechanical theory, finding agreement [Gao et al. (2010); Cheng et al. (2011)]. A thorough review of traditional rheology techniques may be found in the work of Barnes et al. (1989). Transient flows also give insight into the micromechanics of rate-dependent processes in steady-state flow behavior: Sudden removal of external forcing demonstrates that the microstructure relaxes over multiple time scales, each associated with distinct physical processes [Mackay and Kaffashi (1995); Watanabe et al. (1996a, 1996b); Kaffashi et al. (1997); Foss (1999); Zia and Brady (2013)]. Such temporal response reveals the underlying connection between structure and rheology.

Time-dependent behavior is a hallmark of non-Newtonian fluids. Such materials can display both liquidlike (viscous) and solidlike (elastic) behaviors, depending on the rate with which they are perturbed relative to the relaxation time scale(s) of the microstructure. Linear viscoelastic properties are typically studied by imposing a small-amplitude oscillatory shearing motion on a bulk sample of material, $\gamma(t)=\gamma_{0} \sin (\omega t)$, with amplitude $\gamma_{0}$ and oscillation frequency $\omega$ say, where $\dot{\gamma} \equiv \partial \gamma / \partial t$ [Pipkin (1986)]. In this linear-response regime, the resultant shear stress $\sigma(t)$ is linear in the imposed strain and strain rate, with coefficients that form the real and imaginary parts of a complex modulus. The phase shift and amplitude change with respect to strain rate or strain are thus related in a simple way to the storage (elastic modulus) and loss (viscous modulus) of energy in the sheared material. The degree to which each of these mechanisms dominates is encoded into the frequency dependence of the complex moduli. Viscoelastic behavior is found even in systems as simple as a colloidal dispersion of hard spheres. In their 
simplicity, colloidal dispersions make an excellent model system for understanding the microstructural origins of viscoelasticity [Lionberger and Russel (1994); Brady (1993b); Foss (1999); Khair and Brady (2005)]. In theoretical approaches, the suspension stress (and viscosity) can be separated into solvent and particle phase contributions, and the particle-phase stress further resolved into hydrodynamic, interparticle-force, and entropic contributions [Batchelor (1972); Brady (1993a, 1993b); Morris and Brady (1996)].

Oscillatory rheology thus provides insight into flow- and frequency-dependent material behavior. Although many studies focus on small departures from equilibrium, materials of interest are often driven far from equilibrium. For example, the mucus that lines the lungs and airway provides optimal healthy function with both liquidlike and elastic behavior. Strong flows, e.g., coughing, ideally produce viscous flow. But when the elastic modulus dominates the behavior of airway mucus, serious (sometimes life-threatening) pathology results, for example, in the case of cystic fibrosis [App et al. (1998); Banerjee et al. (2001); Lai et al. (2009); Wüstneck et al. (2002)].

There is an ongoing effort to meld two experimental approaches-small amplitude oscillatory shear and steady shear-into one technique, so-called large amplitude oscillatory shear, or LAOS [see, e.g., Dealy and Wissbrun (1990); Giacomin and Dealy (1993); Hyun et al. (2002); Ewoldt et al. (2008)]. In such an approach, a rheometer imposes a steady oscillatory simple-shear of arbitrary amplitude and frequency, and the time-dependent shear stress is measured. Here, the strain is denoted $\gamma(t)=\gamma_{0} \sin (\omega t)$ and the rate of strain is denoted $\dot{\gamma}(t)=\dot{\gamma}_{0} \cos (\omega t)$ with $\dot{\gamma}_{0}=\omega \gamma$, where $\gamma_{0}$ is the maximum strain amplitude. The goal of the experiment is to extract nonlinear viscoelastic properties from the resultant stress signal. A number of approaches to interpreting this stress response have been proposed [Wilhelm (2002); Cho et al. (2005); Ewoldt et al. (2008); Rogers and Lettinga (2012)]. However, such interpretations provide only qualitative indications of nonlinear material behavior or test-specific properties, e.g., shear thinning and thickening or strain softening and hardening that may or may not translate to material behavior in other time-dependent nonlinear deformations. While in linear-response the complex moduli can be transformed to model any small-amplitude time-dependent flow, no such generic procedure appears possible in the nonlinear regime. An alternative is to fit constitutive models to LAOS data and interpret material behavior through the model parameters [Wilhelm (2002); Gurnon and Wagner (2012)], which has proven quite useful in understanding macroscopic behavior but still leaves open the question of its connection to the underlying microstructure. Recent advances in metrology enable direct measurement of the microstructure during LAOS [Cheng et al. (2011); López-Barrón et al. (2012); Rogers et al. (2012)], thereby providing direct microstructural interpretation of the nonlinear stress response.

A tangentially related aspect of the LAOS experiments is an ambiguity in the control parameters, evident in the oscillatory signals noted above. Oscillatory shear is described by just two parameters: The strain amplitude, $\gamma_{0}$, and the oscillation frequency, $\omega$. However, in any shearing experiment, it is natural to consider the strain-rate amplitude, $\dot{\gamma}_{0}$, as well. On one hand, the steady-shear experiment is replicated when the oscillation frequency $\omega$ is small relative to all other rate processes in the material. One thus has, alongside the large strain inherent in the steady-shear regime, a strain rate of arbitrary magnitude. On the other hand, elasticlike materials are highly sensitive to strain amplitude but less sensitive to strain-rate amplitude (in the linearly elastic regime). Finally, frequency sweeps are the preferred method for extracting time-dependent viscoelasticity of a material. In its most powerful form, the LAOS experiment must live on a manifold of strain amplitude $\gamma_{0}$, strain-rate amplitude $\dot{\gamma}_{0}$, and frequency $\omega$. Which is the best projection for a particular experiment? In the present study, we show that the equations governing the microstructural deformation provide unambiguous guidance. 
As noted above, one limitation of past approaches is their limited ability to relate microstructural deformation to macroscopic response. To overcome these limitations, we take a micromechanical approach to form a direct connection between the microstructure under arbitrary strain-rate amplitude and oscillation frequency, and the corresponding stress response.

An additional limitation of such approaches is the macroscopic scale over which material is probed in traditional macroscale shear and LAOS experiments. In such experiments, a bulk shearing motion is imposed on a macroscopic sample of material and the spatially averaged response of the material is thus probed. However, there is strong demand to interrogate small-scale systems such as rare biological fluids or expensive pharmaceutical preparations. Traditional rheology approaches require at least milliliters of fluid which, due to expense or microscale variation, may exclude many important microscale systems.

An alternative is microrheology in which the motion of a colloidal probe suspended in some viscoelastic material is used to infer its linear and nonlinear mechanical properties. Microrheology has august origins-Einstein (1906) and Perrin (1909) used the diffusion of colloidal particles in materials with known viscoelastic properties to measure Avagadro's number. With advances in microscopy and other techniques [see, e.g., Crocker and Grier (1996)], microrheology has become highly valued as a tool for interrogation of complex fluids [Mason and Weitz (1995); MacKintosh and Schmidt (1999); Habdas et al. (2004); Meyer et al. (2006); Squires and Brady (2005); Khair and Brady (2006); Wilson et al. (2009)]. In passive microrheology, thermal fluctuations drive the Brownian movement of colloidal particles. Their mean-squared displacement is tracked over time and related to the viscoelastic moduli of the material in which they are embedded through the generalized Stokes-Einstein-Sutherland equation. A wide range of materials have been studied via microrheology, including actin networks [Gisler and Weitz (1999); Ziemann et al. (1994)], gelatin [Freundlich and Seifriz (1923)], DNA solutions [Mason et al. (1997)], and colloidal dispersions [Crocker (1997); Crocker et al. (2000); Levine and Lubensky (2000); Habdas et al. (2004)]. Microrheology has also been developed as a tool for high-throughput material screening [Breedveld and Pine (2003); Schultz and Furst (2011)].

However, to probe the nonlinear response of a viscoelastic material, it must be driven out of equilibrium through active forcing of the colloidal probes. For instance, optical tweezers and magnetic fields can be used to force probes and produce large deformations in the embedding material. There have been many investigations, both theoretical and experimental, of steady and transient active microrheology [Squires and Brady (2005); Khair and Brady (2005, 2006); Meyer et al. (2006); Wilson et al. (2009); Zia and Brady (2010, 2012, 2013)]. These demonstrate that active microrheology is capable of revealing many of the same physical processes as result from macroscopic deformation.

In nonlinear microrheology, the motion of the probe distorts the surrounding microstructure. At steady state, the degree of this distortion is captured by the Péclet number, $\mathrm{Pe}=F^{\mathrm{ext}} a /(2 k T)$, where $F^{\mathrm{ext}}$ is the strength of external forcing and $2 k T / a$ is the entropic restoring force of the bath due to the Brownian motion of the suspension. Here, $k T$ is the thermal energy and $a$ is the radius of a bath particle. This is the microrheological equivalent of the Weissenberg number used to characterize flows of viscoelastic materials. It is a dimensionless rate of deformation. For an oscillating external force, the viscoelastic response also depends a dimensionless frequency $\alpha=\omega a^{2} / D$, where $D=k T / 6 \pi \eta a$ is the Stokes-Einstein-Sutherland diffusivity. This is the micorheological equivalent of the Deborah number used to characterize oscillatory flows of many viscoelastic materials.

In the present investigation, we study microstructural response to variations in both the strength of forcing, Pe, and the rate of oscillation, $\alpha$, each of arbitrary magnitude. As 
with LAOS, in oscillatory, active microrheology, there are only two independent parameters to vary: Pe and $\alpha$. However, the ratio of forcing strength to oscillation rate, $\mathrm{Pe} / \alpha$, is important as well. This ratio is equivalent to the strain amplitude and we will refer to it throughout the article as the extent of deformation.

To illustrate this phase space pictorially, Fig. 1 gives a map of the response as a function of strength of forcing and rate of oscillation. The three regimes studied in this work are as follows: Linear-response ( $\mathrm{Pe} \ll 1$ or $\alpha \gg 1$ and $\mathrm{Pe} / \alpha \ll 1$ ); steady, nonlinear response (Pe $\gg 1$ and $\alpha \ll 1$ ); and the region of large-amplitude oscillatory forcing (Pe $\gg 1, \alpha \gg 1$, and $\mathrm{Pe} / \alpha \gg 1)$. This last region is of particular interest because, as shall be shown, it reveals a new regime of viscoelastic behavior which we call hypoviscous.

Additionally, we demonstrate through solution of the microrheology problem and physical arguments that the first harmonic of the oscillatory response (whether from microrheology or macrorheology) is the only harmonic whose real and imaginary parts can be termed viscous and elastic unambiguously. The first harmonic appears to generate over $95 \%$ of the response "signal" as the oscillatory process approaches the low frequency limit, and its variation with changes in deformation rate and oscillation rate are at least characteristic of what is seen in the other harmonics. Since the first harmonic will always be the strongest signal measured in a rheology experiment, we suggest that this may provide the most useful characterization of materials. In particular, we feel the first harmonic is the only necessary quantitative descriptor of the deformation process. Measurement of this quantity via a sweep of frequency and amplitude in a LAOS experiment should provide sufficient data for parameter estimation in a constitutive model. The quality of the constitutive model should be tested directly by computing the higher harmonics that result from such an estimate and then comparing them to those from the LAOS experiments.

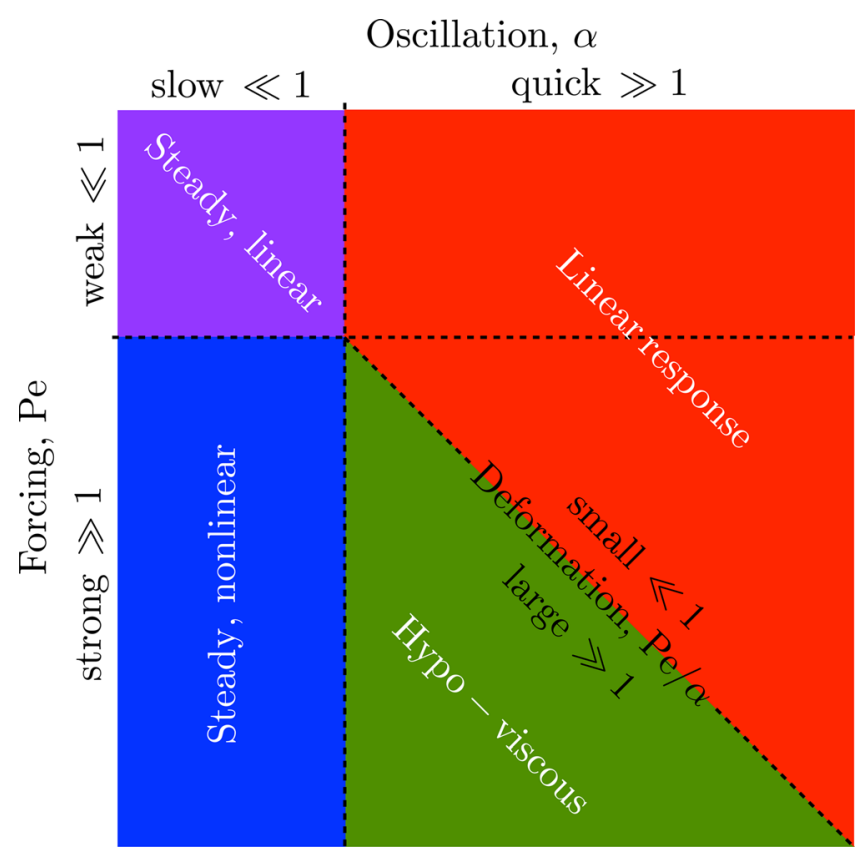

FIG. 1. Probe response phase space. These are four distinct regimes for colloidal dispersions undergoing large amplitude oscillatory deformation via microrheology: The region of steady linear response for which $\mathrm{Pe}, \alpha \ll 1$, the region of linear response for which $\mathrm{Pe} / \alpha \ll 1$, the region of steady, nonlinear response for which $\mathrm{Pe} \gg 1, \alpha \ll 1$ and the hypoviscous regime where $\mathrm{Pe}, \alpha, \mathrm{Pe} / \alpha \gg 1$. 
The remainder of this paper is organized as follows: In Sec. II, the model system for oscillatory microrheology is introduced. Next, in Sec. III, the microstructural view of colloidal dispersions is described in detail and the time-dependent Smoluchowski equation derived. To make analytical progress, an assumption of diluteness is made. This yields the pair-Smoluchowski equation that governs microstructural dynamics. For largeamplitude flows, an expansion of the microstructural deformation as a Fourier series reveals a fundamental aspect of such flows: The corresponding microstructural harmonics are linearly independent but not orthogonal. This leads to a coupling between harmonics that in turn gives rise to a rich interplay between diffusion and advection in determining the microstructure. Following Squires and Brady (2005), an interpretation of Stokes' drag law is then made in Sec. IV to deduce the microvelocity increment-the change in probe velocity due to its interactions with the microstructure. A careful examination of the hydrodynamic, Brownian, and interparticle contributions to the total velocity is conducted to reveal their individual connections to nonlinear viscoelastic behavior. The study is concluded in Sec. VI with a discussion and future outlook.

\section{MODEL COLLOIDAL DISPERSION}

The model system comprises a dispersion of neutrally buoyant colloidal particles with hydrodynamic radius $a_{h}$ immersed in a Newtonian solvent of viscosity $\eta$ and density $\rho$. A probe particle with the same radius is driven by an external force through the suspension. The relative strength of inertial to viscous forces defines a Reynolds number, $\operatorname{Re}=\rho U a_{h} / \eta$, where $U$ is the characteristic probe velocity. Because the probe and bath particles are small, $\operatorname{Re} \ll 1$, inertia can be neglected and the fluid mechanics are governed by Stokes' equations. In the dilute limit, the number density of bath particles, $n_{\text {bath }}$, is small. We treat the particles as hard spheres with a hard-core radius $a$ (see Fig. 2) such that the interparticle potential $V(r)$ as a function of the separation between the particles, $r$, is

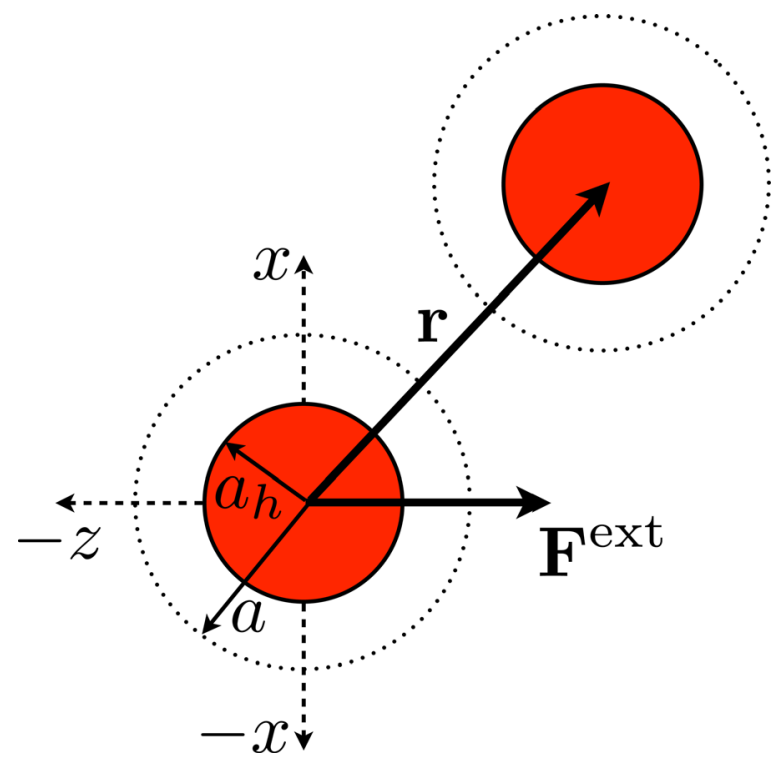

FIG. 2. The suspension is modeled in the dilute limit by considering the interactions between two particles: A probe particle driven by an external force $\mathbf{F}^{\text {ext }}$ and a bath particle that is free of external forcing. Both particles have the same hydrodynamic radius, $a_{h}$, and interact via hard-sphere repulsion when the separation between their centers is $2 a$. 


$$
V(r)= \begin{cases}\infty, & r<2 a \\ 0, & r>2 a\end{cases}
$$

One can envision two limits: One in which the hard-core radius is identical to the hydrodynamic radius, $a=a_{h}$, and another in which the hard-core radius is much larger than the hydrodynamic radius $a \gg a_{h}$. The ratio of the two, $\lambda=a / a_{h}$, has as its limiting cases $\lambda=1$ and $\lambda \rightarrow \infty$, respectively. This interparticle potential derives from the excluded annulus model first employed by Morris and Brady (1996). Squires and Brady (2005) studied the steady behavior in the limit $\lambda \rightarrow \infty$, where hydrodynamic interactions are negligible. Khair and Brady (2006) studied the steady microrheology of colloidal dispersions over a continuous range of $\lambda$. As in that work, the volume fraction is defined with respect to the hard-sphere repulsion, $\phi=4 \pi a^{3} n_{\text {bath }} / 3$. In this study, we focus on the limit of $\lambda=1$ because it offers a complete microstructural model of a viscoelastic material.

As a probe particle is driven through a suspension by a steady external force, the bath particles move in response-via hydrodynamic or hard-sphere interactions. The particles cannot interpenetrate so there is a buildup of bath particles in front of the advancing probe and a deficit trailing it. Figure 3 gives an illustration of the statistical distribution of bath particles, or microstructure, around the probe at steady-state as the external force on the probe is increased. In this depiction, there is a spherical region in which bath particle centers are excluded entropically. This is indicated in black. The concentration of bath particle centers surrounding the probe is indicated by the colors: Red, suggesting higher concentration relative to equilibrium; blue, suggesting lower concentration relative to equilibrium; and green, suggesting a concentration near equilibrium. Throughout this manuscript, we discuss the probability of finding a bath particle a given distance from the probe relative to that probability at equilibrium. This difference is the salient quantity for calculating rheological functions. The growing asymmetry of the microstructure with increasing applied force is characteristic of the transition from a linear to a nonlinear rheological response.

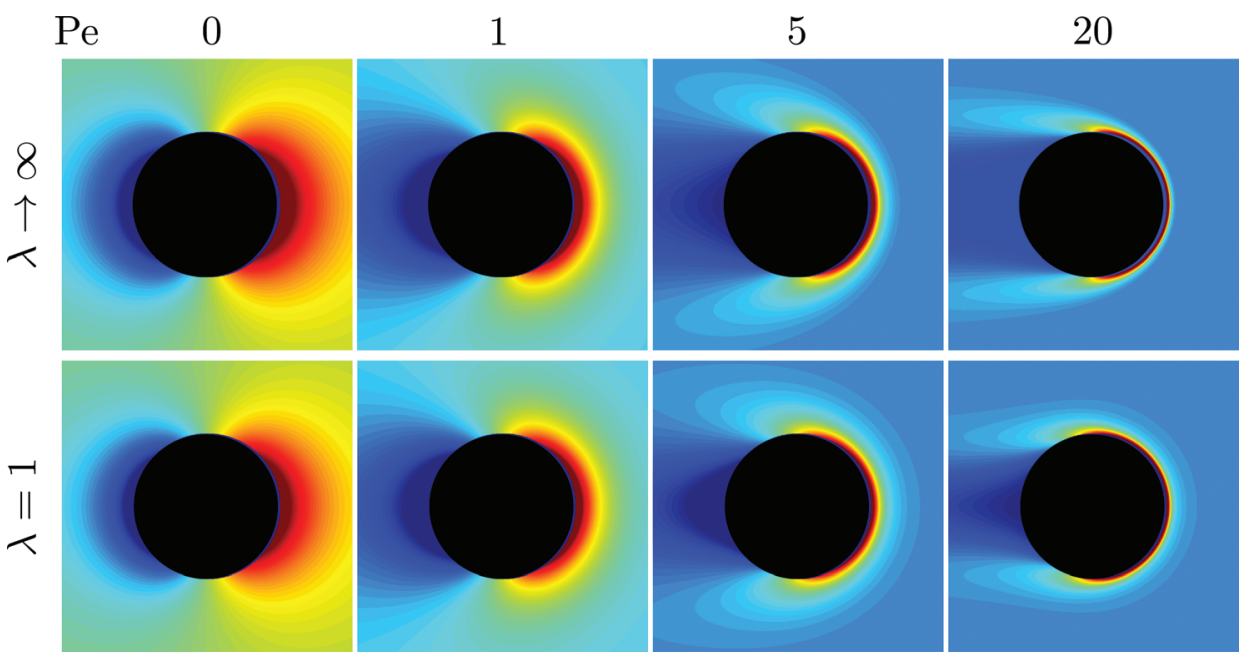

FIG. 3. In a dilute colloidal dispersion, the motion of a probe particle distorts the statistical distribution of the bath particles surrounding it. Here, the probe particle is moving to the right and there is a build-up of bath particles in front (red) and a deficit (dark blue) behind. The force driving the probe through the suspension increases in each image from left to right. The top row shows the structure when hydrodynamic interactions are negligibly weak, as from Squires and Brady (2005), while the bottom row includes hydrodynamic interactions, as from Khair and Brady (2006). 


\section{A MICROSTRUCTURAL VIEW OF LARGE AMPLITUDE OSCILLATION}

In Secs. III A and III B, the equations governing the evolving distribution of particles are presented, along with the appropriate boundary conditions. The development is kept sufficiently general so as to easily extend to any flow of a dilute dispersion of hard spheres. While the theory is developed and solved in the dilute limit, its applicability to suspensions of any concentration is discussed in the Appendix. Most importantly, because the structure of the solution is the same in the $\mathrm{N}$-particle limit, we anticipate a quantitative change, but not a qualitative change, in the flow curves relative to the dilute limit calculation —at least within the liquid regime.

\section{A. The pair Smoluchowski equation}

In the dilute limit, the mechanics of a colloidal dispersion may be represented by the interactions of just two particles with centers at $\mathbf{x}_{1}$ and $\mathbf{x}_{2}$ whose spatiotemporal statistical distribution, $P_{2}\left(\mathbf{x}_{1}, \mathbf{x}_{2}, t\right)$, is governed by the pair Smoluchowski equation

$$
\dot{P}_{2}+\nabla_{1} \cdot \mathbf{j}_{1}+\nabla_{2} \cdot \mathbf{j}_{2}=0,
$$

where $\mathbf{j}_{1}$ and $\mathbf{j}_{2}$ are the flux of particles 1 and 2 due to external, interparticle, and Brownian forces and $\dot{P}$ is the time rate-of-change of the particle distribution. In this case, the particles interact via a hard-sphere repulsive potential and particle 1 is driven by an external force $\mathbf{F}^{\text {ext }}$. The fluxes of particles 1 and 2 are then

$$
\mathbf{j}_{1}=\mathbf{U}_{1} P_{2}=\frac{1}{k T} \mathbf{D}_{11} \cdot \mathbf{F}^{\text {ext }} P_{2}-\left(\mathbf{D}_{11} \cdot \nabla_{1}+\mathbf{D}_{12} \cdot \nabla_{2}\right) P_{2}
$$

and

$$
\mathbf{j}_{2}=\mathbf{U}_{2} P_{2}=\frac{1}{k T} \mathbf{D}_{21} \cdot \mathbf{F}^{\text {ext }} P_{2}-\left(\mathbf{D}_{21} \cdot \nabla_{1}+\mathbf{D}_{22} \cdot \nabla_{2}\right) P_{2} .
$$

Here, $\mathbf{D}_{\alpha \beta}$ is the diffusion tensor coupling the force on particle $\beta$ to the motion of particle $\alpha$ via hydrodynamic interactions. The diffusion tensors for a pair of particles are well known [Batchelor (1976); Jeffrey and Onishi (1984); Kim and Karrila (1991)]. They depend only on the interparticle spacing and are independent of the absolute position of the particles, suggesting a coordinate transformation: $\mathbf{r} \equiv \mathbf{x}_{2}-\mathbf{x}_{1}, \mathbf{z} \equiv \mathbf{x}_{1}$ such that

$$
\dot{P}_{2}+\nabla_{z} \cdot \mathbf{j}_{1}+\nabla_{r} \cdot\left(\mathbf{j}_{2}-\mathbf{j}_{1}\right)=0 .
$$

Integrating over $\mathbf{z}$ while holding $\mathbf{r}$ fixed gives an equation for the conditional probability of finding a bath particle a distance $\mathbf{r}$ from the probe, $P_{1 \mid 1}(\mathbf{r} \mid \mathbf{z}, t)$. The conditional probability is linearly proportional to the pair distribution function, $P_{1 \mid 1}(\mathbf{r} \mid \mathbf{z}, t)=n_{\text {bath }} g(\mathbf{r}, t)$, so that the Smoluchowski equation becomes

$$
\dot{g}+\frac{1}{n_{\text {bath }}} \nabla_{r} \cdot\left(\int\left(\mathbf{j}_{2}-\mathbf{j}_{1}\right) d \mathbf{z}\right)=0 .
$$

The relative diffusivity is given by $\mathbf{D}_{r}=\mathbf{D}_{22}+\mathbf{D}_{11}-\mathbf{D}_{12}-\mathbf{D}_{21}$. Since the particles are equal in size, $\mathbf{D}_{11}-\mathbf{D}_{21}=\mathbf{D}_{r} / 2$ and the relative flux is given by

$$
\frac{1}{n_{\text {bath }}} \int\left(\mathbf{j}_{2}-\mathbf{j}_{1}\right) d \mathbf{z}=\frac{1}{2 k T} \mathbf{D}_{r} \cdot \mathbf{F}^{\text {ext }} g-\mathbf{D}_{r} \cdot \nabla_{r} g,
$$


and the pair Smoluchowski equation simplifies to

$$
\dot{g}=\nabla_{r} \cdot\left[\mathbf{D}_{r} \cdot\left(-\frac{1}{2 k T} \mathbf{F}^{\mathrm{ext}} g+\nabla_{r} g\right)\right] .
$$

There is no long-range order: $g(\mathbf{r}, t) \sim 1$, at infinite separation since the conditional probability of finding a bath particle a distance $\mathbf{r}$ from the probe at $\mathbf{z}$ should be $n_{\text {bath }}$ in the dilute limit. The relative flux is zero at interparticle contact

$$
\hat{\mathbf{r}} \cdot\left(-\frac{1}{2 k T} \mathbf{D}_{r} \cdot \mathbf{F}^{\mathrm{ext}} g-\mathbf{D}_{r} \cdot \nabla_{r} g\right)=0 \quad \text { at } \quad r=2 a .
$$

In this study, the external force on the probe particle is fixed in magnitude and oscillates in time, $\mathbf{F}^{\text {ext }}=\hat{\mathbf{F}}^{\text {ext }} \cos (\omega t)$. It is important to note that by beginning with the Smoluchowski equation as a model for the particle distribution, we have assumed that momentum relaxation of the particles is orders of magnitude faster than any other dynamical process in the suspension. This is an excellent approximation for colloids at equilibrium. The introduction of a new rate process, oscillatory forcing with frequency $\omega$, into the Smoluchowski equation is straightforward with the restriction that $\omega$ is much smaller than the momentum relaxation rate too. With the substitution $\mathbf{D}_{r} \cdot \hat{\mathbf{F}}^{\text {ext }} /(2 k T) \rightarrow \mathbf{U}$, the pair Smoluchowski equation may then be expressed in a form that is valid for any relative velocity field, $\mathbf{U}$, depending only on $\mathbf{r}$, for instance a shear flow

$$
\dot{g}=\nabla_{r} \cdot\left(-\mathbf{U} \cos (\omega t) g+\mathbf{D}_{r} \cdot \nabla_{r} g\right),
$$

with $g(\mathbf{r}, t)=1$ as $r \rightarrow \infty$ and the no-flux condition at interparticle contact

$$
\hat{\mathbf{r}} \cdot\left(-\mathbf{U} \cos (\omega t) g+\mathbf{D}_{r} \cdot \nabla_{r} g\right)=0 .
$$

To gain insight into the effects of the relative strengths of advective, diffusive, and oscillatory motion, the next step is to simplify the Smoluchowski equations via dimensional analysis.

\section{B. Coupling of structural modes}

The structure of Smoluchowski equation (10) is equally applicable to other colloidal flows, for instance, particles in simple shear. Only the form of the relative velocity, $\mathbf{U}$, changes. Likewise, and as illustrated in the Appendix, the following Fourier decomposition of the suspension microstructure is hardly limited to the dilute limit. Thus, in what follows, we offer general view of how the microstructure in a suspension of hard-spheres evolves when driven by an oscillation of arbitrary amplitude.

The Smoluchowski equation can be simplified through dimensional analysis by scaling the separation between particles on the hard-core radius, $a$, the relative velocity on its maximum amplitude, $U$, the relative diffusivity on its value at infinite separation, $D$, and time on the diffusive time scale, $a^{2} / D$. This gives rise to two dimensionless groups: The Péclet number, which characterizes the strength of the force deforming the material, $\mathrm{Pe}=U a / D$; and $\alpha=\omega a^{2} / D$, a dimensionless frequency that characterizes the oscillation rate relative the thermal relaxation time scale of the material. The values of $U$ and $D$ applicable to microrheology are discussed in Sec. IV. To focus on departures from equilibrium, we express the pair distribution function as $g(\mathbf{r}, t)=1+\operatorname{Pe} f(\mathbf{r}, t)$ where $f(\mathbf{r}, t)$ represents the deviation of $g(\mathbf{r}, t)$ from the equilibrium value of unity. When made dimensionless in this way, the Smoluchowski equation is 


$$
\dot{f}=\nabla_{r} \cdot\left[-\mathbf{U} \cos (\alpha t)(1+\operatorname{Pe} f)+\mathbf{D}_{r} \cdot \nabla_{r} f\right],
$$

with $f=0$ as $r \rightarrow \infty$, and the no-flux condition at interparticle contact $r=2$ is

$$
\hat{\mathbf{r}} \cdot\left[\mathbf{U} \cos (\alpha t)(1+\mathrm{Pe} f)-\mathbf{D}_{r} \cdot \nabla_{r} f\right]=0 .
$$

In Eqs. (12) and (13), all quantities, including the velocity $\mathbf{U}$ and diffusivity $\mathbf{D}_{r}$, have been made dimensionless by the scalings noted above. Unless otherwise indicated, they remain dimensionless throughout the rest of this study.

We propose a solution to Eqs. (12) and (13) as a Fourier series in harmonics of the base frequency $\alpha$

$$
f(\mathbf{r}, t)=\sum_{n=-\infty}^{\infty} e^{i n \alpha t} f_{n}(\mathbf{r})
$$

Substitution into the Smoluchowski equation leads to governing equations for the Fourier coefficients, $f_{n}(\mathbf{r})$,

$$
\operatorname{in\alpha f_{n}}=\nabla_{r} \cdot\left[-\frac{1}{2} \mathbf{U}\left(\delta_{1 n}+\delta_{-1 n}+\operatorname{Pe}\left(f_{n+1}+f_{n-1}\right)\right)+\mathbf{D}_{r} \cdot \nabla_{r} f_{n}\right],
$$

where $\delta_{i j}$ is the Kronecker delta function. The far-field boundary condition is $f_{n}=0$ as $r$ $\rightarrow \infty$, and the no-flux condition at $r=2$ is

$$
\hat{\mathbf{r}} \cdot\left[\frac{1}{2} \mathbf{U}\left(\delta_{1 n}+\delta_{-1 n}+\operatorname{Pe}\left(f_{n+1}+f_{n-1}\right)\right)-\mathbf{D}_{r} \cdot \nabla_{r} f_{n}\right]=0 .
$$

Each temporal mode $f_{n}(\mathbf{r})$ is coupled to $f_{n+1}(\mathbf{r})$ and $f_{n-1}(\mathbf{r})$ by the oscillatory advection. The coupling strength is set by the Péclet number, an important consequence of large amplitude oscillatory flow. Higher harmonics of the microstructural deformation are inherently connected. This plays a central role all of the themes explored in this work.

The doubly infinite series 14 can be simplified by recognizing that the microstructural perturbation, $f(\mathbf{r}, t)$, is a real-valued function. The modes $f_{n}(\mathbf{r})$ and $f_{-n}(\mathbf{r})$ are related by their complex conjugate, $f_{n}(\mathbf{r})=\hat{f}_{-n}(\mathbf{r})$, where the hat symbol denotes the complex conjugate. In consequence, the microstructural perturbation can be determined by solving for $n \geq 0$ alone.

To understand the scaling with respect to Pe of each harmonic, one may examine individually governing equation (15) for $n=0,1,2$

$$
\begin{gathered}
0=\nabla_{r} \cdot\left[-\frac{\operatorname{Pe}}{2} \mathbf{U}\left(f_{1}+\hat{f}_{1}\right)+\mathbf{D}_{r} \cdot \nabla_{r} f_{0}\right], \\
i \alpha f_{1}=\nabla_{r} \cdot\left[-\frac{1}{2} \mathbf{U}\left(1+\operatorname{Pe}\left(f_{2}+f_{0}\right)\right)+\mathbf{D}_{r} \cdot \nabla_{r} f_{1}\right], \\
2 i \alpha f_{2}=\nabla_{r} \cdot\left[-\frac{\operatorname{Pe}}{2} \mathbf{U}\left(f_{3}+f_{1}\right)+\mathbf{D}_{r} \cdot \nabla_{r} f_{2}\right] .
\end{gathered}
$$

It is apparent that in the limit of weak forcing, $\mathrm{Pe} \ll 1$, the Fourier coefficients scale as $f_{ \pm 1} \sim O(1), f_{0}, f_{ \pm 2} \sim O(\mathrm{Pe}), f_{ \pm 3} \sim O\left(\mathrm{Pe}^{2}\right)$. By induction, we conclude that the leading 
order contribution to mode $f_{n}(\mathbf{r})$ scales as Pe $\mathrm{e}^{|| n|-1|}$. This important result holds regardless of how the suspension is deformed when $\mathrm{Pe} \ll 1$. In the limit of weak deformation, the temporal modes decompose into a simple hierarchy of equations with each mode coupled to a neighboring mode by a small factor, Pe. When the deformation is not weak, however, the harmonics must be solved simultaneously instead.

\section{NONLINEAR MICROVISCOELASTICITY}

One goal of rheology is to connect average flow properties of a complex fluid to the deformation of its microstructure. Up to now, the microstructural model describes the evolution of a dilute colloidal suspension subject to an arbitrary flow. In this section, we address one specific flow that due to single-particle forcing and the resulting microrheology. We begin with a brief review of the expressions for microviscosity following Squires and Brady (2005) and Khair and Brady (2006). These expressions can then be combined with the equations governing the microstructure presented in Sec. III to obtain nonlinear viscoelastic properties of a colloidal dispersion.

There are two primary modes of probe motion in microrheology: One in which the probe moves under a fixed imposed force and the other in which its velocity is constrained. In the former mode, the probe is driven through the suspension by a fixed (and in this case oscillatory) force. The probe particle thus moves with its Stokes velocity, less a hindrance factor related to its interactions with the suspension. The Stokes velocity gives the characteristic magnitude of probe motion, $U=F^{\mathrm{ext}} /\left(6 \pi \eta a_{h}\right)$. Both the probe particle and the bath particles can diffuse. Their relative diffusivity sets the characteristic diffusive scale, $D=k T /\left(3 \pi \eta a_{h}\right)$. Together the external force magnitude and relative diffusivity set the relative strength and rate of microstructural deformation, respectively, defining a Péclet number $\mathrm{Pe}=F^{\mathrm{ext}} a /(2 k T)$ and dimensionless frequency $\alpha=3 \pi \eta a_{h} a^{2} \omega /(k T)$.

The relative velocity and diffusivity made dimensionless by these scalings are

$$
\mathbf{U}=\mathbf{D} \cdot \hat{\mathbf{F}}^{\mathrm{ext}}, \quad \mathbf{D}=G(r) \hat{\mathbf{r}} \hat{\mathbf{r}}+H(r)(\mathbf{I}-\hat{\mathbf{r}} \hat{\mathbf{r}})
$$

where $G(r)$ and $H(r)$ are scalar hydrodynamic functions characterizing the magnitude of the relative diffusion along and normal to the line connecting the centers of the probe and another particle in the suspension. That is, $G(r)$ and $H(r)$ are the orthogonal components of the relative diffusion tensor, $\mathbf{D}_{r}$, normalized by the characteristic diffusive scale, $k T /\left(3 \pi \eta a_{h}\right)$ [Batchelor and Green (1972); Kim and Karrila (1991); Khair and Brady (2006)].

\section{A. A review of active microrheology}

The velocity $\mathbf{U}_{1}$ of a probe particle translating under a fixed-force, $\mathbf{F}^{\text {ext }}$, alone through an otherwise quiescent solvent is given by Stokes' drag law. The probe velocity, $\mathbf{U}_{1}=\mathbf{U}^{\text {Stokes }}=\mathbf{F}^{\text {ext }} /\left(6 \pi \eta a_{h}\right)$, is proportional to the applied force and inversely proportional to its hydrodynamic radius $a_{h}$ and the solvent viscosity $\eta$. The probe velocity is thus a measure of the solvent viscosity. When the probe is driven through a suspension of other particles, the microstructure slows the probe's motion. Squires and Brady (2005) interpreted this reduction in mean probe velocity, $\left\langle\mathbf{U}_{1}\right\rangle$, as a drag due to the solvent and bath particles, and defined an effective viscosity, $\eta^{\text {eff }}$, via analogy to Stokes' drag law 


$$
\mathbf{F}^{\mathrm{ext}}=6 \pi \eta^{\mathrm{eff}} a_{h}\left\langle\mathbf{U}_{1}\right\rangle
$$

The angle brackets $\langle\cdot\rangle$ signify an ensemble average where the probable arrangements of bath particles around the probe are given by the pair distribution function $g(\mathbf{r}, t)$. The effective viscosity is thus

$$
\frac{\eta^{\mathrm{eff}}}{\eta}=\left(\frac{\mathbf{F}^{\mathrm{ext}} \cdot \mathbf{F}^{\mathrm{ext}}}{6 \pi \eta a_{h}}\right) /\left(\left\langle\mathbf{U}_{1}\right\rangle \cdot \mathbf{F}^{\mathrm{ext}}\right) .
$$

The effective viscosity may be separated into the contributions due to the solvent and that due to the particles

$$
\frac{\eta^{\mathrm{eff}}}{\eta}=1+\frac{\eta^{\mathrm{micro}}}{\eta},
$$

where the microviscosity $\eta^{\text {micro }} / \eta$ characterizes drag due to the bath particles-above and beyond the solvent drag. The average probe velocity $\left\langle\mathbf{U}_{1}\right\rangle$ is what one measures in experiments to determine the viscosity of the suspension.

There are two microstructural contributions to the increased effective viscosity: The rigidity of individual particles and the distribution of those particles relative to each other. The first aspect, the nondeformability of the particles and the no-slip condition at their surface, leads to distorted fluid streamlines and increasing viscous dissipation-a hydrodynamic effect. However, the overall arrangement of particles is deformable as well. This has interesting and deformation-rate-dependent consequences for the viscosity. Rate-dependent viscosity is a hallmark of non-Newtonian behaviors such as shear thinning and shear thickening. In the context of microrheology, to understand the origin of this rate-dependent response, one must examine how hydrodynamic, interparticle, and Brownian forces reduce the average probe velocity, $\left\langle\mathbf{U}_{1}\right\rangle$. The contributions can be classified as either conservative or nonconservative, as follows.

Conservative forces-Brownian and interparticle, in the present case-slow the probe's motion. These two contributions, denoted $\left\langle\mathbf{U}^{B}\right\rangle$ and $\left\langle\mathbf{U}^{P}\right\rangle$, respectively, are each proportional to the deformed microstructure $f(\mathbf{r}, t)$ and hence vanish at equilibrium. The Brownian force can be understood in the following way: The motion of the probe deforms the distribution of bath particles from equilibrium. This is a reduction in entropy that is countered by a thermodynamic driving force to make uniform the bath particle distribution. This force opposes the external force on the probe, thus increasing viscous resistance. Interparticle forces can arise from a variety of colloidal-scale interactions (e.g., electrostatic, depletion, or dispersion forces). Just as the Brownian force acts to drive the particles toward an entropic maximum, interparticle forces drive the microstructure toward the configuration of minimum potential energy. Departures from equilibrium thus produce an analogous restoring force that resists probe motion. By definition, the conservative forces sum to zero at equilibrium, and this balance of conservative forces is characteristic of the Boltzmann distribution: $g(r) \sim e^{-V(r) / k T}$. Note, we term the Brownian forces "conservative" because from the perspective of the Smoluchowski equation they can be written as the gradient of a potential, for instance $k T \log g(r)$. However, conservative force between particles can enhance the rate of viscous dissipation in a dispersion.

In contrast, hydrodynamic forces are nonconservative. The reduction in probe speed due to hydrodynamic forces is proportional to the imposed force: $\left\langle\mathbf{U}^{H}\right\rangle=\langle\mathbf{M}\rangle \cdot \mathbf{F}^{\text {ext }}$, where $\mathbf{M}$ is a mobility matrix that depends only on the configuration of the particles. In 
the simplest case of a single particle alone in a solvent, $\mathbf{M}=\mathbf{I} / 6 \pi \eta \mathrm{a}$. Even in an equilibrium configuration, the particles interact hydrodynamically. For instance, a probe diffusing passively in such a dispersion is hindered even over short time scales by the presence of the other particles. The corresponding reduction in probe velocity is $\left\langle\mathbf{U}_{\infty}^{H}\right\rangle$ (the superscript $\infty$ indicates that this corresponds to the high-frequency viscosity.)

The average probe velocity includes contributions due to hydrodynamic, interparticle, and Brownian interactions: $\left\langle\mathbf{U}_{1}\right\rangle=\mathbf{U}^{\text {Stokes }}+\left\langle\mathbf{U}^{H}\right\rangle+\left\langle\mathbf{U}^{P}\right\rangle+\left\langle\mathbf{U}^{B}\right\rangle$. In the dilute limit, there are corresponding hydrodynamic, interparticle, and Brownian contributions to the microviscosity: $\eta^{\text {micro }}=\eta^{H}+\eta^{P}+\eta^{B}$ [Squires and Brady (2005); Khair and Brady (2006)]. Because the hydrodynamic velocity is linear in the imposed force, $\left\langle\mathbf{U}^{H}\right\rangle$ vanishes instantly upon removal of the external force. In contradistinction, the Brownian force decays to zero over a finite time after removal of the external force. That is, the microstructural perturbation $f(\mathbf{r}, t)$ relaxes gradually as the Brownian motion of the particles homogenizes their distribution. The time scale for this relaxation is diffusive, $6 \pi \eta a_{h}^{3} / k T$. This gradual relaxation has been interpreted previously as a release of free energy that was stored in the microstructure via work done by the probe to distort the particle microstructure [e.g., Zia and Brady (2013) among others]. The notion of entropic energy storage is an important one and plays a central role in the remainder of the paper.

We saw in Sec. III that non-Newtonian microrheology is encoded in the deformed microstructure $f(\mathbf{r}, t)$ [cf. Eq. (8) and Fig. 3] and inferred from the mean probe velocity [cf. Eqs. (20) and (21)]. To examine the effects of only the deformed microstructure on the velocity-above and beyond that due to the solvent, $\mathbf{U}^{\text {Stokes }}$, and that due to the equilibrium microstructure, $\mathbf{U}_{\infty}^{H}$-we define the microvelocity

$$
\left\langle\mathbf{U}^{\text {micro }}\right\rangle \equiv\left\langle\mathbf{U}_{1}\right\rangle-\mathbf{U}^{\text {Stokes }}-\left\langle\mathbf{U}_{\infty}^{H}\right\rangle .
$$

In Eq. (22), we have removed the contributions to the probe's velocity resulting from its Stokes drag in the solvent, $\mathbf{U}^{\text {Stokes }}$, and hydrodynamic interactions through an undeformed microstructure, $\left\langle\mathbf{U}_{\infty}^{H}\right\rangle$. Thus the microvelocity, $\left\langle\mathbf{U}^{\text {micro }}\right\rangle$, reveals how distortion of the microstructure affects probe motion-in particular, the rates at which the suspension stores and dissipates the work done on it.

Thus far, we have discussed the motion of a probe driven by a fixed external force and the corresponding deformation of the microstructure through which it moves. Alternatively, one could drive the probe at a fixed (oscillatory) velocity, $\mathbf{U}_{1}$. Fixed-force experiments are typically carried out by driving the probe with magnetic tweezers [Habdas et al. (2004)]. In fixed-velocity experiments, the probe can be held in a stiff optical trap while the bath is moved past it at a fixed rate [Meyer et al. (2006)]. ${ }^{1}$ Interesting dynamical differences between the two modes arise. In the fixed-force case, the probe velocity fluctuates as it moves through the bath. As a result, the probe experiences a collision-induced diffusive spread of its trajectory [Zia and Brady (2010)]. In the fixed-velocity case, however, the probe's motion is prescribed and so it cannot diffuse. In our recent work, we showed that hydrodynamic interactions cause dissimilarities in the microstructural evolution and hence in the microviscosity [Swan and Zia (2013)].

\footnotetext{
${ }^{1}$ The idea of a fixed force or fixed velocity is an approximation, the accuracy of which is dictated by the uniformity of the applied field or the stiffness of the optical trap. Such approximations can be made quite accurate [Habdas et al. (2004); Meyer et al. (2006)].
} 
A fixed-velocity analogue for the effective viscosity can be made via Stokes' drag law, following the procedure given above for the fixed-force case: $\left\langle\mathbf{F}^{\mathrm{ext}}\right\rangle=6 \pi \eta^{\mathrm{eff}} a_{h} \mathbf{U}_{1}$. As with Eq. (20), the effective viscosity in the fixed-velocity case is

$$
\frac{\eta^{\text {eff }}}{\eta}=\left(\left\langle\mathbf{F}^{\text {ext }}\right\rangle \cdot \mathbf{U}_{1}\right) /\left(6 \pi \eta a_{h} \mathbf{U}_{1} \cdot \mathbf{U}_{1}\right) .
$$

Here, the external force required to drive the particle with a fixed velocity may be decomposed into hydrodynamic, interparticle, and Brownian contributions: $\left\langle\mathbf{F}^{\text {ext }}\right\rangle=\mathbf{F}^{\text {Stokes }}$ $+\left\langle\mathbf{F}^{H}\right\rangle+\left\langle\mathbf{F}^{P}\right\rangle+\left\langle\mathbf{F}^{B}\right\rangle$, where $\mathbf{F}^{\text {Stokes }}=6 \pi \eta a_{h} \mathbf{U}_{1}$. The microviscosity measured in the fixed-velocity mode is thus

$$
\frac{\eta^{\text {micro }}}{\eta}=\frac{\eta^{\text {eff }}}{\eta}-1=\frac{\left(\left\langle\mathbf{F}^{H}\right\rangle+\left\langle\mathbf{F}^{P}\right\rangle+\left\langle\mathbf{F}^{B}\right\rangle\right) \cdot \mathbf{U}_{1}}{6 \pi \eta a_{h} \mathbf{U}_{1} \cdot \mathbf{U}_{1}} .
$$

As with the fixed-force mode, there is a contribution to the external force on the probe particle that depends only on the deformed suspension microstructure- the microforce

$$
\left\langle\mathbf{F}^{\text {micro }}\right\rangle \equiv\left\langle\mathbf{F}^{\text {ext }}\right\rangle-\mathbf{F}^{\text {Stokes }}-\left\langle\mathbf{F}_{\infty}^{H}\right\rangle,
$$

where $\left\langle\mathbf{F}_{\infty}^{H}\right\rangle$ is the additional force on the probe particle due to dragging of the solvent through a suspension in its equilibrium configuration.

We have completed the analysis of large amplitude oscillatory microrheology in both the fixed-force and the fixed-velocity limits and find no qualitative differences. However, the fixed-force case is pedagogically and phenomenologically more straightforward than the fixed-velocity case. For clarity, we focus on the former throughout the rest of this study.

\section{B. Probe velocity in response to fixed amplitude, oscillatory external force}

In addition to Stokes drag, three suspension-mediated mechanisms hinder probe motion: First, hydrodynamic interactions with the bath particles; second, resistance due to gradients in bath particle concentration (entropic forces); and third, interparticle forces. Their formulation has been given explicitly by Khair and Brady (2006) for a dilute suspension with particles of equal size

$$
\begin{aligned}
\frac{6 \pi \eta a_{h}}{F^{\mathrm{ext}}}\left\langle\Delta \mathbf{U}^{H}\right\rangle=\frac{3 \phi}{4 \pi} \operatorname{Pe} \cos (\alpha t) \hat{\mathbf{F}}^{\mathrm{ext}} \cdot \int_{r \geq 2}\left[A_{11}(r) \hat{\mathbf{r}} \hat{\mathbf{r}}+B_{11}(r)(\mathbf{I}-\hat{\mathbf{r}} \hat{\mathbf{r}})-\mathbf{I}\right] f(\mathbf{r}, t) d \mathbf{r}, \\
\frac{6 \pi \eta a_{h}}{F^{\mathrm{ext}}}\left\langle\mathbf{U}^{B}\right\rangle=-\frac{3 \phi}{8 \pi} \int_{r \geq 2} W(r) f(\mathbf{r}, t) \hat{\mathbf{r}} d \mathbf{r} \\
\frac{6 \pi \eta a_{h}}{F^{\mathrm{ext}}}\left\langle\mathbf{U}^{P}\right\rangle=-\frac{3 \phi}{2 \pi} G(2) \int_{r=2} f(\mathbf{r}, t) \hat{\mathbf{r}} d \Omega
\end{aligned}
$$

where $\phi=4 \pi a^{3} n_{\text {bath }} / 3$ is the volume fraction of the bath particles based on the hardcore radius. The scalar hydrodynamic functions, $A_{11}(r)$ and $B_{11}(r)$, are the orthogonal components of the diffusion tensor $\mathbf{D}_{11}$ normalized by the Stokes-Einstein diffusivity, $k T /(6 \pi \eta a)$. In the absence of hydrodynamic interactions, they take on the values $A_{11}(r)=B_{11}(r)=1$. With hydrodynamic interactions, these scale as $1+O\left(r^{-4}\right)$ so that 
the hydrodynamic integral in Eq. (26a) is absolutely convergent. The function $W(r)$ arises from the gradient of the relative diffusion tensor and represents the Brownian drift driven by variation in the statistical distribution of bath particles. It is defined as $W(r)$ $=d_{r} G(r)+(2 / r)(G(r)-H(r))$ and is zero without hydrodynamic interactions.

In Eq. (26a), we have defined the hydrodynamic contribution to probe hindrance that is due strictly to the deformed microstructure, $\left\langle\Delta \mathbf{U}^{H}\right\rangle \equiv\left\langle\mathbf{U}^{H}\right\rangle-\left\langle\mathbf{U}_{\infty}^{H}\right\rangle$. Together the three contributions to probe hindrance given by Eqs. (26a)-(26c) form the total microstructural reduction in probe velocity [cf. Eq. (22)]

$$
\left\langle\mathbf{U}^{\text {micro }}\right\rangle=\left\langle\Delta \mathbf{U}^{\mathrm{H}}\right\rangle+\left\langle\mathbf{U}^{\mathrm{B}}\right\rangle+\left\langle\mathbf{U}^{\mathrm{P}}\right\rangle .
$$

The hindrance due to the equilibrium structure in Eq. (22) is given by

$$
\left\langle\mathbf{U}_{\infty}^{H}\right\rangle=-\phi \cos (\alpha t) U_{\infty}^{H} \hat{\mathbf{F}}^{\mathrm{ext}}
$$

where $U_{\infty}^{H}$ is defined in terms of the scalar hydrodynamic functions as

$$
U_{\infty}^{H}=-\int_{2}^{\infty}\left(A_{11}(r)+2 B_{11}(r)-3\right) r^{2} d r
$$

so that $U_{\infty}^{H}=1.828$ when $\lambda=1$ and is zero in the absence of hydrodynamic interactions. The quantity $U_{\infty}^{H}=\left(1-6 \pi \eta a D_{0}^{S} / k T\right) / \phi$ is the $O(\phi)$ decrement in the short-time selfdiffusivity for a colloidal particle in a hard-sphere dispersion. It is important to note that the hindrance due to hydrodynamic interactions possesses a "phase factor" $\cos (\alpha t)$ lacking in the other contributions [those being linear in $f(\mathbf{r}, t)$ alone]. Consequently, one expects a different temporal character for the hydrodynamic response to large amplitude oscillatory perturbation. This is made clear upon expressing each of these contributions as a Fourier series. Recall that the goal is to connect the velocity with the structural modes (Fourier coefficients) of $f(\mathbf{r}, t)$ [cf. Eq. (15) in Sec. III B)

$$
\frac{6 \pi \eta a_{h}}{F^{\mathrm{ext}}}\langle\mathbf{X}\rangle=-\phi\left(\sum_{n=-\infty}^{\infty} e^{i n \alpha t} \mathbf{X}_{n}\right),
$$

with $\mathbf{X}=\Delta \mathbf{U}^{H}, \mathbf{U}^{B}, \mathbf{U}^{P}$. The harmonics are

$$
\begin{aligned}
\Delta \mathbf{U}_{n}^{H}=-\frac{3}{8 \pi} \operatorname{Pe} \hat{\mathbf{F}}^{\mathrm{ext}} \cdot \int_{r \geq 2}\left[A_{11}(r) \hat{\mathbf{r}} \hat{\mathbf{r}}+B_{11}(r)(\mathbf{I}-\hat{\mathbf{r}} \hat{\mathbf{r}})-\mathbf{I}\right]\left[f_{n-1}(\mathbf{r})+f_{n+1}(\mathbf{r})\right] d \mathbf{r} \\
\mathbf{U}_{n}^{B}=\frac{3}{8 \pi} \int_{r \geq 2} W(r) f_{n}(\mathbf{r}) \hat{\mathbf{r}} d \mathbf{r} \\
\mathbf{U}_{n}^{P}=\frac{3}{2 \pi} G(2) \int_{r=2} f_{n}(\mathbf{r}) \hat{\mathbf{r}} d \Omega
\end{aligned}
$$

A key result of this analysis is that the slowing of the probe due to conservative forcesas temporal modes $\mathbf{U}_{n}^{B}$ and $\mathbf{U}_{n}^{P}$-is proportional to the microstructural mode $f_{n}(\mathbf{r})$. The slowing of the probe due to hydrodynamic forces is different, however. The temporal mode $\mathbf{U}_{n}^{H}$ is linear in $f_{n-1}(\mathbf{r})+f_{n+1}(\mathbf{r})$ instead. This will have a profound effect on how the conservative and nonconservative forces change the probe velocity. In particular, the 
hydrodynamic forces will not contribute to measures of cycle averaged elasticity. They are dissipative!

Equations (31a)-(31c) confirm the well-known result that hydrodynamic forces propagated through the deformed microstructure play no role in linear-response [Russel et al. (1989); Brady (1993b); Khair and Brady (2005)]. Linear-response derives exclusively from the first harmonic. As shown in Sec. III B, when Pe $\ll 1$, the leading contribution to the distorted microstructure, $f_{ \pm 1}(\mathbf{r})$ is $O(1)$. Indeed, the first effects of the structure on $\mathbf{U}_{1}^{H}$ are linear in $f_{0}(\mathbf{r})+f_{2}(\mathbf{r})$ which scale as Pe and thus are quadratic in the Péclet number.

Thus far, we have expressed each of the contributions to the probe velocity as a sum of harmonics that correspond to temporal oscillations, each weighting the corresponding microstructural mode $f_{n}(\mathbf{r})$. It remains now obtain the microstructural modes by solving Smoluchowski equation (15).

\section{Solution methodologies}

The governing equations for the structural harmonics [Eqs. (15) and (16)] in spherical polar coordinates $(r, \theta)$ with the polar axis parallel to $\hat{\mathbf{F}}^{\mathrm{ext}}$ are

$$
\begin{aligned}
& \frac{1}{2} \operatorname{Pe}\left[\cos \theta G(r) \partial_{r}\left(f_{1}+\hat{f}_{1}\right)-\frac{\sin \theta}{r} H(r) \partial_{\theta}\left(f_{1}+\hat{f}_{1}\right)\right] \\
& \quad+\operatorname{Pe} \cos \theta W(r)\left(f_{1}+\hat{f}_{1}\right)=\frac{1}{r^{2}} \partial_{r}\left(r^{2} G(r) \partial_{r} f_{0}\right)+\frac{H(r)}{r^{2} \sin \theta} \partial_{\theta}\left(\sin \theta \partial_{\theta} f_{0}\right),
\end{aligned}
$$

with boundary condition at $r=2$

$$
\frac{1}{2} \cos \theta \mathrm{Pe}\left(f_{1}+\hat{f}_{1}\right)-\partial_{r} f_{0}=0
$$

and for $n \geq 1$

$$
\begin{aligned}
& \operatorname{in} \alpha f_{n}+\frac{1}{2} \operatorname{Pe}\left\{\cos \theta\left[G(r) \partial_{r}\left(f_{n-1}+f_{n+1}\right)+W(r)\left(f_{n-1}+f_{n+1}\right)\right]-\frac{\sin \theta}{r} H(r) \partial_{\theta}\left(f_{n-1}+f_{n+1}\right)\right\} \\
& \quad=\frac{1}{r^{2}} \partial_{r}\left(r^{2} G(r) \partial_{r} f_{n}\right)+\frac{H(r)}{r^{2} \sin \theta} \partial_{\theta}\left(\sin \theta \partial_{\theta} f_{n}\right)-\frac{1}{2} \cos \theta W(r) \delta_{1 n},
\end{aligned}
$$

with boundary condition at $r=2$

$$
\frac{1}{2} \cos \theta\left[\delta_{1 n}+\operatorname{Pe}\left(f_{n-1}+f_{n+1}\right)\right]-\partial_{r} f_{n}=0 .
$$

Two approaches are taken here to solve the governing equations. First, an expansion in Legendre polynomials yields important insight into the time-reversal and spatial symmetry of the deformed microstructure. To explore a larger parameter space, a numerical solution is more convenient. In both cases, the coupling between the harmonics is truncated at 20 terms, achieved by enforcing the constraint: $f_{21}(\mathbf{r}) \equiv 0$. The accuracy of this truncation is tested explicitly in Sec. V A 3.

\section{Legendre polynomial expansion}

We solve for the microstructural functions using the Ansatz 


$$
f_{n}=\sum_{j=0}^{\infty} R_{n}^{(j)}(r) P_{j}(\cos \theta),
$$

where $R_{n}^{(j)}(r)$ are the radial solutions to be determined, and $P_{j}(\cos \theta)$ are the Legendre polynomials of degree $j$. We then substitute this expansion into Eqs. (32a) and (32b) governing $f_{0}$, and into Eqs. (33a) and (33b) for $f_{n}$ with $n \geq 1$. Capitalizing on the orthogonality of the Legendre polynomials results in a system of ordinary differential equations for the unknown functions of $r$

$$
\begin{aligned}
& G(r) \ddot{R}_{0}^{(j)}+\left(W(r)+\frac{2}{r} H(r)\right) \dot{R}_{0}^{(j)}-\frac{j(j+1)}{r^{2}} H(r) R_{0}^{(j)} \\
&= \frac{1}{2} \operatorname{Pe}\left[\alpha_{j}\left(\dot{R}_{1}^{(j-1)}+\dot{\hat{R}}_{1}^{(j-1)}\right)+\beta_{j}\left(R_{1}^{(j-1)}+\hat{R}_{1}^{(j-1)}\right)\right. \\
&\left.\quad+\gamma_{j}\left(\dot{R}_{1}^{(j+1)}+\dot{\hat{R}}_{1}^{(j+1)}\right)+\delta_{j}\left(R_{1}^{(j-1)}+\hat{R}_{1}^{(j-1)}\right)\right],
\end{aligned}
$$

with boundary conditions

$$
\begin{gathered}
\dot{R}_{0}^{(j)}=\frac{1}{2} \operatorname{Pe}\left[\frac{j}{2 j-1}\left(R_{1}^{(j-1)}+\hat{R}_{1}^{(j-1)}\right)+\frac{j+1}{2 j+3}\left(R_{1}^{(j+1)}+\hat{R}_{1}^{(j+1)}\right)\right], \quad r=2 \\
R_{0}^{(j)} \rightarrow 0 \quad r \rightarrow \infty,
\end{gathered}
$$

and for $n>1$

$$
\begin{aligned}
G(r) & \ddot{R}_{n}^{(j)}+\left(W(r)+\frac{2}{r} H(r)\right) \dot{R}_{n}^{(j)}-\left[\frac{j(j+1)}{r^{2}} H(r)+i n \alpha\right] R_{n}^{(j)} \\
= & \frac{1}{2} W \delta_{1 n} \delta_{j 1}+\frac{1}{2} \operatorname{Pe}\left[\alpha_{j}\left(\dot{R}_{n-1}^{(j-1)}+\dot{R}_{n+1}^{(j-1)}\right)+\beta_{j}\left(R_{n-1}^{(j-1)}+R_{n+1}^{(j-1)}\right)\right. \\
& \left.+\gamma_{j}\left(\dot{R}_{n-1}^{(j+1)}+\dot{R}_{n+1}^{(j+1)}\right)+\delta_{j}\left(R_{n-1}^{(j+1)}+R_{n+1}^{(j+1)}\right)\right],
\end{aligned}
$$

with boundary conditions

$$
\begin{gathered}
\dot{R}_{n}^{(j)}=\frac{1}{2} \delta_{1 n} \delta_{j 1}+\frac{1}{2} \operatorname{Pe}\left[\frac{j}{2 j-1}\left(R_{n-1}^{(j-1)}+R_{n+1}^{(j-1)}\right)+\frac{j+1}{2 j+3}\left(R_{n-1}^{(j+1)}+R_{n+1}^{(j+1)}\right)\right], \quad r=2 \\
R_{n}^{(j)} \rightarrow 0 \quad r \rightarrow \infty .
\end{gathered}
$$

In these equations, the Greek coefficients are defined as

$$
\begin{aligned}
\alpha_{j} & =\frac{j}{2 j-1} G(r), & \beta_{j} & =\frac{j}{2 j-1}\left(W(r)-\frac{j-1}{r} H(r)\right), \\
\gamma_{j} & =\frac{j+1}{2 j+3} G(r), & \delta_{j} & =\frac{j+1}{2 j+3}\left(W(r)+\frac{j+2}{r} H(r)\right) .
\end{aligned}
$$

This spectral decomposition reveals a number of interesting features of the perturbed microstructure and its impact on probe motion. First, we note that the only inhomogeneity 
in Eqs. (35) and (38) arises from the terms proportional to $\delta_{1 n \delta_{\mathrm{j}}}$, which is the odd Legendre mode of an odd Fourier mode. A closer look shows that the odd-odd, even-even pairs are coupled through the right-hand side of each equation (e.g., terms like $R_{n-1}^{(j-1)}$ or $R_{n+1}^{(j+1)}$ ). Physically, the forcing propagates through these structural modes. The odd-even and even-odd modes form their own coupled structure, but it is homogeneous. Therefore, we may conclude that $R_{2 n}^{(2 j+1)} \equiv R_{2 n+1}^{(2 j)} \equiv 0$, reducing the computational burden by half. But far more importantly, this structure underlies the time-reversal symmetry required in low-Reynolds number flows. This will be discussed in more detail in Sec. IV C 2. Inserting these expressions for the microstructure into the integrals defining the micro velocity components obtains

$$
\begin{aligned}
\Delta \mathbf{U}_{n}^{H}= & -\frac{1}{2} \operatorname{Pe}^{\text {ext }} \int_{r \geq 2}\left[\frac{2}{5}\left(A_{11}(r)-B_{11}(r)\right)\left(R_{n-1}^{(2)}+R_{n+1}^{(2)}\right)+\left(A_{11}(r)+2 B_{11}(r)-3\right)\right. \\
& \left.\times\left(R_{n-1}^{(0)}+R_{n+1}^{(0)}\right)\right] r^{2} d r, \\
\mathbf{U}_{n}^{B}= & \frac{1}{2} \hat{\mathbf{F}}^{\text {ext }} \int_{r \geq 2} W(r) R_{n}^{(1)} r^{2} d r \\
\mathbf{U}_{n}^{P}= & 2 G(2) R_{n}^{(1)}(2) \hat{\mathbf{F}}^{\mathrm{ext}} .
\end{aligned}
$$

We may thus conclude, without loss of generality and through appeal only to the suspension structure, that

$$
\mathbf{U}_{2 n}^{H} \equiv \mathbf{U}_{2 n}^{B} \equiv \mathbf{U}_{2 n}^{P} \equiv 0
$$

That is, there are no even-numbered Fourier modes of the microvelocity increment. This preserves the time reversal symmetry of the probe particle's response. Conversely, any mechanism that would break the symmetry of $f_{0}$ would lead to nonzero, even numbered Fourier modes of the displacement (e.g., if the external force applied to the probe has a nonzero temporal mean).

We choose a large but finite number of Legendre modes $\left(j_{\max }\right)$ beyond which the series in Eq. (34) is truncated and determine the $R_{2 n}^{(2 j)}$ and $R_{2 n+1}^{(2 j+1)}$ for $n \leq 20$. These equations are solved simultaneously using the MATLAB routine $b v p 4 c$ which features adaptive grid refinement and error control. The sources of error are then set by truncation chosen for the Legendre polynomial and Fourier series. For Pe $\leq 10$ and $\alpha=0$, one needs fewer than 60 Legendre polynomials to recover the well-known steady-state solutions to within $0.001 \%$. Similarly, for $\mathrm{Pe}=0$ and $\alpha>0$, the linear response is recovered exactly utilizing only two harmonics and the first two Legendre polynomials. We thus anticipate that solution of these equations is practicable for all values of $\alpha$ and $\mathrm{Pe} \leq 10$ with $j_{\max }=60$. In favor of computational efficiency, fewer may be chosen and the truncation level tested for convergence.

\section{Finite-difference solution}

For larger Péclet numbers (Pe > 10), the structural harmonics [Eqs. (15) and (16)] are solved using a finite difference approximation with an orthogonal grid in spherical polar coordinates. The grid points are distributed uniformly in both $\theta$ and the (scaled) radial coordinate $y=\exp [-\max (\mathrm{Pe}, 1)(r-2)]$. As noted in Sec. IV C 1, odd/even harmonics of the microstructural perturbation depend only on odd/even Legendre polynomials of $\cos \theta$. The odd and even harmonics must satisfy the pair of conditions $\partial_{\theta} f_{n}=0$ ( $n$ odd) and $f_{n}=0$ ( $n$ even) at $\theta=0$ and $f_{n}=0(n$ odd $)$ and $\partial_{\theta} f_{n}=0(n$ even $)$ at $\theta=\pi / 2$. The axisymmetric governing equations are thus solved only on the quarter-plane, $y \in[0,1)$ 
and $\theta \in[0, \pi / 2]$ rather than over the full half plane, reducing the computational burden by half as noted above. Upon discretization, a point in the computational domain is labeled $\left(y_{i}, \theta_{j}\right)$; the $n$th harmonic of the microstructural perturbation at that point is labeled $f_{n}^{(i, j)}$. Here, the indices $(i, j)$ span $(0,0)$ to $(M, N)$ so that the uniform discretization is given in terms of $(\Delta y, \Delta \theta)=(1 / N, \pi / 2 M)$ for which $\left(y_{i}, \theta_{j}\right)=(i \Delta y, j \Delta \theta)$. The forcing terms proportional to $-W(r) \cos \theta\left(\delta_{n 1}+\delta_{-1 n}\right)$ are similarly discretized.

Applying a second-order central differencing stencil in $y$ and $\theta$ to the partial differential equations (35)-(38) yields

$$
\begin{aligned}
& A_{n}^{(i, j)} f_{n}^{(i-1, j)}+B_{n}^{(i, j)} f_{n}^{(i, j-1)}+C_{n}^{(i, j)} f_{n}^{(i, j)}+D_{n}^{(i, j)} f_{n}^{(i, j+1)}+E_{n}^{(i, j)} f_{n}^{(i+1, j)} \\
& \quad+\alpha_{n}^{(i, j)}\left(f_{n-1}^{(i-1, j)}-f_{n-1}^{(i+1, j)}+f_{n+1}^{(i-1, j)}-f_{n+1}^{(i+1, j)}\right)+\beta_{n}^{(i, j)}\left(f_{n-1}^{(i, j-1)}-f_{n-1}^{(i, j+1)}+f_{n+1}^{(i, j-1)}-f_{n+1}^{(i, j+1)}\right) \\
& \quad+\gamma_{n}^{(i, j)}\left(f_{n-1}^{(i, j)}+f_{n+1}^{(i, j)}\right)=b_{n}^{(i, j)}
\end{aligned}
$$

where the $(i, j, n)$ th coefficients take on the particular values below given $y=y_{i}$, $r=2-\log \left(y_{i}\right) / \max (\mathrm{Pe}, 1)$, and $\theta=\theta_{j}$

$$
\begin{aligned}
& A_{n}^{(i, j)}=G(r)\left[\left(\frac{d y}{d r}\right)^{2}\left(\frac{1}{\Delta y^{2}}\right)-\left(\frac{d^{2} y}{d r^{2}}+\frac{2}{r} \frac{d y}{d r}\right)\left(\frac{1}{2 \Delta y}\right)\right]-\frac{1}{r^{2}} \frac{d G(r)}{d r}\left(\frac{d y}{d r}\right)\left(\frac{1}{2 \Delta y}\right), \\
& B_{n}^{(i, j)}=\frac{H(r)}{r^{2}}\left[\left(\frac{1}{\Delta \theta^{2}}\right)-\cot \theta\left(\frac{1}{2 \Delta \theta}\right)\right], \\
& C_{n}^{(i, j)}=-G(r)\left(\frac{d y}{d r}\right)^{2}\left(\frac{2}{\Delta y^{2}}\right)-\frac{H(r)}{r^{2}}\left(\frac{2}{\Delta \theta^{2}}\right)-i n \alpha, \\
& D_{n}^{(i, j)}=\frac{H(r)}{r^{2}}\left[\left(\frac{1}{\Delta \theta^{2}}\right)+\cot \theta\left(\frac{1}{2 \Delta \theta}\right)\right], \\
& E_{n}^{(i, j)}=G(r)\left[\left(\frac{d y}{d r}\right)^{2}\left(\frac{1}{\Delta y^{2}}\right)+\left(\frac{d^{2} y}{d r^{2}}+\frac{2}{r} \frac{d y}{d r}\right)\left(\frac{1}{2 \Delta y}\right)\right]+\frac{1}{r^{2}} \frac{d G(r)}{d r}\left(\frac{d y}{d r}\right)\left(\frac{1}{2 \Delta y}\right), \\
& \alpha_{n}^{(i, j)}=\frac{\operatorname{Pe}}{2} \cos \theta G(r) \frac{d y}{d r}\left(\frac{1}{2 \Delta y}\right), \\
& \beta_{n}^{(i, j)}=-\frac{P e}{2} \sin \theta \frac{H(r)}{r}\left(\frac{1}{2 \Delta \theta}\right), \\
& \gamma_{n}^{(i, j)}=-\frac{\operatorname{Pe}}{2} \cos \theta W(r), \\
& b_{n}^{(i, j)}=\frac{1}{2} \cos \theta W(r)\left(\delta_{1 n}+\delta_{-1 n}\right) .
\end{aligned}
$$

The resulting hierarchy of discretized equations is arranged compactly as $\mathbf{A} \cdot \mathbf{f}=\mathbf{b}$, where $\mathbf{A}$ is a matrix with 15 bands containing the coefficients of the finite difference components, $\mathbf{f}$ is the vector of the solution at all points in the domain, and $\mathbf{b}$ is the vector of forcing at each point in the domain. The boundary conditions define the matrix entries at contact and at infinite separation. The linear system is solved for $\mathbf{f}$ utilizing sparse direct solvers in MATLAB. A discretized domain of 600 grid points in the radial dimension and 300 grid points in the angular dimension with 20 harmonics achieves a relative error of less than $10^{-6}$ for the steady microviscosity $(\alpha=0$ with $\mathrm{Pe} \leq 100)$ as compared to a high accuracy solution of the steady state equations. 


\section{RESULTS}

The solutions of the governing equations presented thus far describe the interactions between a probe driven by a fixed-amplitude oscillatory force in a colloidal dispersion. The role of hydrodynamic forces has been kept quite general by utilizing the excluded annulus model described in Sec. II. The strength of hydrodynamic interactions can vary from negligible $(\lambda \rightarrow \infty)$ to strong $(\lambda \rightarrow 1)$. We focus our discussion here on the latter limit in which hydrodynamic interactions are strongest. In this limit, a pair of particles cannot touch so that there is no hard-sphere force and $\left\langle\mathbf{U}^{P}\right\rangle=0$. Results in the former, freely draining limit will be presented elsewhere.

\section{A. Asymptotic limits}

We begin with a focus on asymptotic behavior in large-amplitude oscillatory microrheology. Three regimes are of particular interest: The linear-response regime $(\mathrm{Pe} \ll 1)$; the high-frequency limit $(\alpha \gg 1)$; and the steady-state response $(\alpha \ll 1)$. Each of these three asymptotic limits is shown pictorially in Fig. 4 as a region of the overall phase space explored (strong to weak forcing/fast to slow oscillation).

\section{Weak forcing $(P e \ll 1)$, linear response}

In the linear-response regime (region 1 in Fig. 4), the force driving deformation of the microstructure is much weaker than the thermal forces on the bath particles, $F^{\text {ext }} \ll k T / a$ or Pe $\ll 1$. Here, the leading order contributions to the Fourier decomposition of the microstructure, $f_{n}(\mathbf{r})$ with $n \neq \pm 1$, are asymptotically small. The modes $f_{ \pm 1}(\mathbf{r})$ are order unity however. The microstructural deformation oscillates sinusoidally with the same frequency as the driving force. The Brownian element of the microvelocity makes the greatest contribution to probe-speed reduction in this limit. The hydrodynamic contribution to the microvelocity is at least linear in the Péclet number and hence is inconsequential in

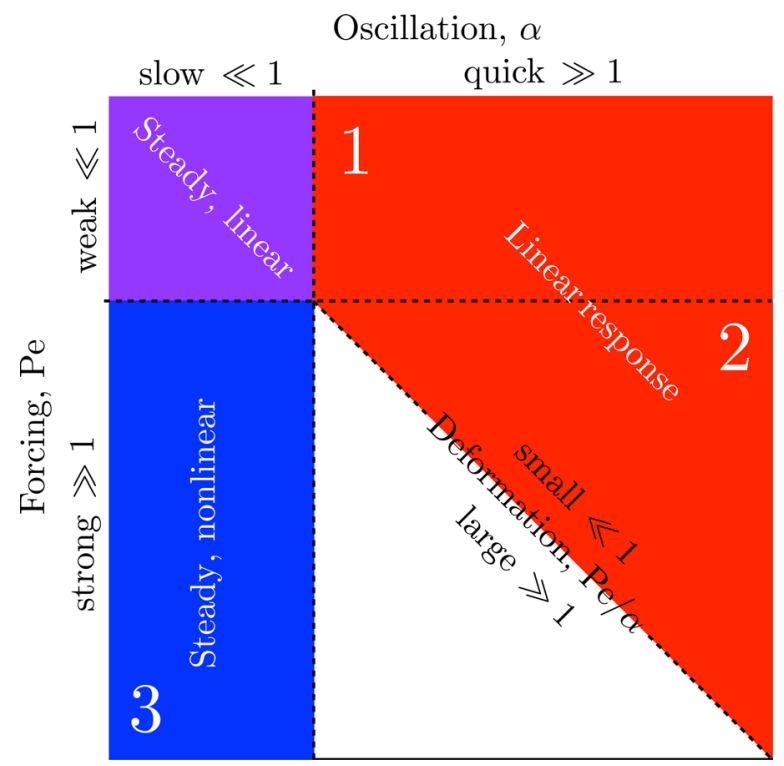

FIG. 4. Three asymptotic limits of forcing strength $(\mathrm{Pe})$ and oscillation rate $(\alpha)$ with numbers indicating relevant domains: (1) Linear response, $\mathrm{Pe} \ll 1$; (2) linear response, $\alpha \gg 1$ and $\mathrm{Pe} / \alpha \ll 1$; (3) steady, nonlinear response, $\alpha \ll 1$. The three limits are studied in Sec. V A 1-V A 3, respectively. 
this limit. Thus, we focus the discussion in the remainder of this section on $n= \pm 1$. To do so, we follow the approach of Khair and Brady (2005) in their analysis of linear viscoelasticity.

The linear-response limit, Pe $\ll 1$, has the solution $f_{ \pm 1}(\mathbf{r})=\Phi_{ \pm 1}(r) \hat{\mathbf{F}}^{\text {ext }} \cdot \hat{\mathbf{r}}$. Insertion of this expression into Eq. (17b) while neglecting terms smaller than $O(1)$ yields

$$
\pm i \alpha \Phi_{ \pm 1}=-\frac{1}{2} W(r)+\frac{1}{r^{2}} \frac{d}{d r}\left(r^{2} G(r) \frac{d}{d r} \Phi_{ \pm 1}\right)-\frac{2}{r^{2}} H(r) \Phi_{ \pm 1},
$$

with $\Phi_{ \pm 1} \rightarrow 0$ as $r \rightarrow \infty$ and $d \Phi_{ \pm 1} / d r=1 / 2$ at $r=2$. The Brownian contribution to the microvelocity is related to the real and imaginary parts of $\Phi_{ \pm 1}(r)$ as

$$
\begin{aligned}
\frac{6 \pi \eta a_{h}}{F^{\mathrm{ext}}}\left\langle\mathbf{U}^{B}\right\rangle= & -\phi\left[\left(\int_{2}^{\infty} W(r) \mathcal{R}\left\{\Phi_{1}(r)\right\} r^{2} d r\right) \cos (\alpha t)\right. \\
& \left.-\left(\int_{2}^{\infty} W(r) \mathcal{I}\left\{\Phi_{1}(r)\right\} r^{2} d r\right) \sin (\alpha t)\right] \hat{\mathbf{F}}^{\mathrm{ext}},
\end{aligned}
$$

where we recognize that the real part is in phase and the imaginary part is out of phase with the external force. This inspires us to write the total microvelocity more generally as

$$
\frac{6 \pi \eta a_{h}}{F^{\mathrm{ext}}}\left\langle\mathbf{U}^{\mathrm{micro}}\right\rangle=\chi^{\prime}(\alpha) \cos (\alpha t)-\chi^{\prime \prime}(\alpha) \sin (\alpha t),
$$

where $\chi^{\prime}(\alpha)$ and $-\chi^{\prime \prime}(\alpha)$ are the real and imaginary parts of the susceptibility $\chi(\alpha)$ relating the microvelocity to the fixed oscillatory force.

The normalized susceptibilities are plotted in Fig. 5. As is typical of viscoelastic media, the out-of-phase component of the susceptibility, $\chi^{\prime \prime}$, grows linearly in $\alpha$ from

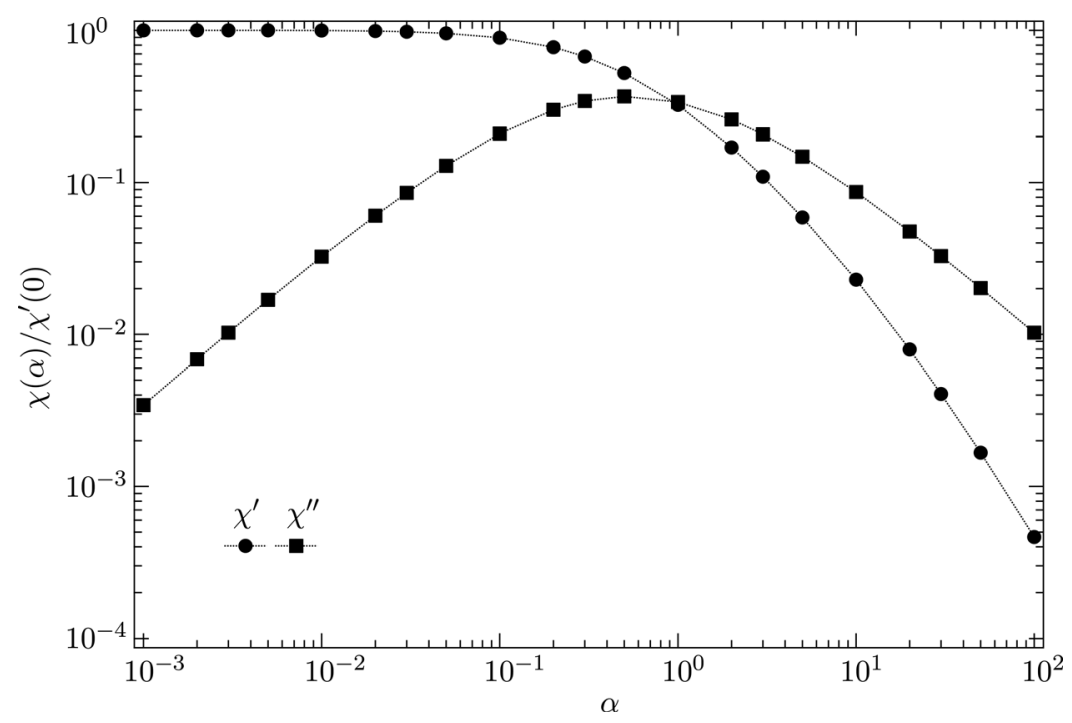

FIG. 5. Linear susceptibility, $\chi$, relating the averaged probe velocity to the imposed external force, as a function of oscillation frequency, $\alpha$. Both real $\left(\chi^{\prime}\right)$ and imaginary $\left(\chi^{\prime \prime}\right)$ parts are shown, each normalized by the value of $\chi^{\prime}$ at $\alpha=0$. 
zero at low frequency, reaches a peak at a frequency near the characteristic relaxation rate of the material, and then decays again as $\alpha^{-1}$ at high frequencies. In contrast, the in-phase component $\chi^{\prime}(\alpha)$ decays as $\alpha^{-2}$ at high frequencies. This demonstrates that at high frequencies, the microdisplacement of the probe particle (the integral in time of the microvelocity) is in-phase with the imposed force and the suspension exhibits elasticlike response.

\section{Rapid oscillation ( $\alpha \gg 1$ ), linear response}

In the high-frequency limit, $\alpha \gg 1$ (region 2 of Fig. 4), the time scale over which oscillation occurs is much shorter than that for thermal relaxation. Over most of the domain, diffusion is relatively slow and the microstructural perturbation is set by the oscillatory force alone. The natural next step is to discard the diffusive terms in Eqs. (32a) and (32b). This produces a leading order scaling of the Fourier modes of the microstructural perturbation with respect to Pe and $\alpha: f_{ \pm 1}(\mathbf{r})=O\left(\alpha^{-1}\right), f_{0}(\mathbf{r})=O\left(\mathrm{Pe} / \alpha^{2}\right)$, and $f_{n}(\mathbf{r}) \sim O\left(\operatorname{Pe} f_{n-1}(\mathbf{r}) / \alpha\right)$ for $n>1$. The largest of these functions, termed outer solutions, is given by

$$
f_{ \pm 1}(\mathbf{r})= \pm \frac{i}{2 \alpha} W(r) \cos \theta
$$

when $\mathrm{Pe} \ll \alpha$. However, as one might anticipate, this solution no longer satisfies the noflux condition at interparticle contact. The higher harmonics fail to satisfy this condition as well. There is a boundary layer near interparticle contact, where diffusion balances the oscillatory force and acts to preserve the no-flux condition. The domain is separated into two regions: An outer region where the oscillation dominates and Eq. (47) is valid and a region inside the boundary layer where diffusion is significant.

When the no-slip surfaces of two spherical particles approach contact, the hydrodynamic force hindering further relative motion diverges as $1 /(r-2)$. Because of this divergence, the thickness of the boundary layer scales as $1 / \alpha$. "Stretching" the radial coordinate inside the boundary layer so that $y=\alpha(r-2)$ is $O(1)$ within it is sufficient to preserve a diffusive term inside the governing equations. In this region, the leading order contributions for $f_{1}(\mathbf{r})$ are

$$
G_{1} \partial_{y}^{2} f_{1}-\frac{1}{2 \alpha} \cos \theta W_{0}=i f_{1},
$$

with $\partial_{y} f_{1}=\cos \theta /(2 \alpha)$ at $y=0$. Here, $W_{0}=W(2)=1.598$ and $G_{1}=G_{\prime}(2)=2$. Clearly, $f_{1}$ is $O\left(\alpha^{-1}\right)$ in the boundary layer and must match the $O\left(\alpha^{-1}\right)$ outer solution as $y \rightarrow \infty$ [Khair and Brady (2005)]. Therefore, over this region

$$
f_{1}(\mathbf{r})=\frac{i}{2 \alpha} W_{0} \cos \theta-\frac{\sqrt{2 G_{1}} W_{0}(1-i)}{4 \alpha} \cos \theta e^{-\sqrt{2 / G_{1}}(1+i) y / 2} .
$$

The leading order contributions to the higher microstructural perturbations $(n>1)$ within the boundary layer satisfy the equation

$$
\inf _{n}+\frac{1}{2}\left(\frac{\mathrm{Pe}}{\alpha}\right)\left[\cos \theta\left(G_{1} \partial_{y} f_{n-1}+W_{0} f_{n-1}\right)-\frac{1}{2} \sin \theta H_{0} \partial_{\theta} f_{n-1}\right]=G_{1} \partial_{y}^{2} f_{n},
$$

with $\partial_{y} f_{n}=(\mathrm{Pe} / \alpha) \cos \theta f_{n-1} / 2$ at $y=0$ and matching to the outer solution as $y \rightarrow \infty$. The quantity $H_{0}=H(2)=0.402$. Therefore, in the boundary layer $f_{n} \sim \mathrm{Pe}^{n-1} / \alpha^{n}$ for $n>1$. 
Again, $f_{1}(\mathbf{r})$ is the biggest contributor so long as $\alpha \gg$ Pe. Recall, however, that when the hydrodynamic and hard-sphere radii are equal, the Brownian contribution to the microvelocity depends on a volume integral of the microstructural perturbation over the range $r>2$. Since the boundary layer is $\alpha^{-1}$ thin, the volume integral over the boundary layer is at least $O\left(\alpha^{-1}\right)$. In consequence, the effect on probe speed due to the perturbation $f_{1}(\mathbf{r})$ in the boundary layer scales as $\alpha^{-2}$. In contrast, the same integral over the outer solution for $f_{1}(\mathbf{r})$ makes an $O\left(\alpha^{-1}\right)$ contribution.

The key point here is that $f_{1}(\mathbf{r})$ makes the biggest contribution to the microstructural perturbation when $\alpha \gg \mathrm{Pe}$, matching the small-force-amplitude solutions of Khair and Brady (2005). That is, when $\alpha \gg P$ e, the motion of the probe particle, and the corresponding distortion of the microstructure, sits squarely in the linear-response regime. This is made more obvious if one recognizes the ratio of the two parameters as the extent of deformation, defined by $\Gamma=\mathrm{Pe} / \alpha$. Thus, $\Gamma \ll 1$ is an alternative definition of linear response to $\mathrm{Pe} \ll 1$. Both are valid. In Fig. 4, all of regions 1 and 2 are thus the linear-response regime, allowing a great collapse of the parameter space.

\section{Slow oscillation $(\alpha \ll 1)$, steady response}

The low-frequency limit ( $\alpha \ll 1$, region 3 in Fig. 4 ) is a particularly interesting one. As oscillation becomes vanishingly slow, it is natural to view the deformation as a timeinvariant steady state. Indeed, the solution of the Smoluchowski equation is unique for a truly steady $(\alpha \equiv 0)$ external force (or velocity)

$$
0=\nabla_{r} \cdot\left[-\mathbf{U}(1+\operatorname{Pe} f)+\mathbf{D}_{r} \cdot \nabla_{r} f\right],
$$

with $f=0$ as $r \rightarrow \infty$, and the no-flux condition at interparticle contact $r=2$ is

$$
\hat{\mathbf{r}} \cdot\left[\mathbf{U}(1+\mathrm{Pe} f)-\mathbf{D}_{r} \cdot \nabla_{r} f\right]=0 .
$$

In the truly steady case, there is no temporal variation. However, there is a different limit in which the rate of oscillation is much slower than any other relaxation process in the dispersion. This suggests that the scaling of time on diffusion is inappropriate. Making time dimensionless on the oscillation rate, $\omega$, captures the physics relevant to slow oscillation. Applying this scaling to the pair-Smoluchowski equation, one obtains the following dimensionless form:

$$
\alpha \dot{f}=\nabla_{r} \cdot\left[-\mathbf{U} \cos \hat{t}(1+\mathrm{Pe} f)+\mathbf{D}_{r} \cdot \nabla_{r} f\right]
$$

with $f=0$ as $r \rightarrow \infty$ and the no-flux condition at interparticle contact $r=2$ is

$$
\hat{\mathbf{r}} \cdot\left[\mathbf{U} \cos \hat{t}(1+\mathrm{Pe} f)-\mathbf{D}_{r} \cdot \nabla_{r} f\right]=0,
$$

where $\hat{t}=\omega t$. If we now allow $\alpha$ to approach zero, $\alpha \dot{f}$ on the left-hand side vanishes but the oscillatory nature of the motion is retained in the advective term on the right-hand side of the equation. This yields a "pseudosteady" limit. At any time $\hat{t}$, the microstructural perturbation $f(\mathbf{r}, \hat{t})$ is essentially independent of its past and future. But since the cosine varies between 1 and -1 , the external force on the probe is constrained to $[-\mathrm{Pe}, \mathrm{Pe}]$. The variation occurs so slowly, however, that from moment to moment, the microstructure instantaneously assumes a new, pseudosteady state corresponding to each new value of $\mathrm{Pe} \cos \hat{t}$. Thus for a given force amplitude Pe, the solution for $f(\mathbf{r}, \hat{t})$ is equivalent to the steady microstructural perturbation $f(\mathbf{r} ; \operatorname{Pe} \cos \hat{t})$. That is, the structure continuously 
and smoothly varies from one steady state to the next, and progression in time simply dictates changes in effective Pe.

Obviously then, the velocity of the probe particle must be pseudosteady as well. This provides a perfect opportunity to test our Fourier series expansion of the Smoluchowski equation against the well-known steady-state solution already described by Khair and Brady (2006). Here, we write the Fourier series for the microstructural perturbation and microvelocity as

$$
f(\mathbf{r}, \hat{t})=\sum_{n=-\infty}^{\infty} e^{i n \hat{t}} f_{n}(\mathbf{r})
$$

and

$$
\frac{6 \pi \eta a_{h}}{F^{\mathrm{ext}}}\left\langle\mathbf{U}^{\mathrm{micro}}\right\rangle=-\phi \sum_{n=-\infty}^{\infty} e^{i n \hat{t}}\left(\Delta \mathbf{U}_{n}^{H}+\mathbf{U}_{n}^{B}+\mathbf{U}_{n}^{P}\right)
$$

The definitions of $\Delta \mathbf{U}_{n}^{H}, \mathbf{U}_{n}^{B}$, and $\mathbf{U}_{n}^{P}$ remain the same as in Eqs. (31a)-(31c). We solve for the $f_{n}(\mathbf{r})$ exactly as described in Sec. IV C with $\alpha=0$ while truncating the series with $n \in[-20,20]$.

In Fig. 6, we plot the reduction in probe speed due to the microstructural deformation, $-\left\langle\mathbf{U}^{\text {micro }}\right\rangle \cdot \hat{\mathbf{F}}^{\text {ext }}\left(3 \pi \eta a_{h}^{2}\right) /(\phi k T)$. It has been normalized by the velocity scale set by the relative diffusion of bath and probe particles, $k T /\left(3 \pi \eta a_{h}^{2}\right)$, and the bath particle volume fraction, $\phi$. We have chosen values for the pseudosteady strength of the external force, Pecost $\hat{t}$, to be the same for all values of Pe. Thus, if we have accurately calculated the

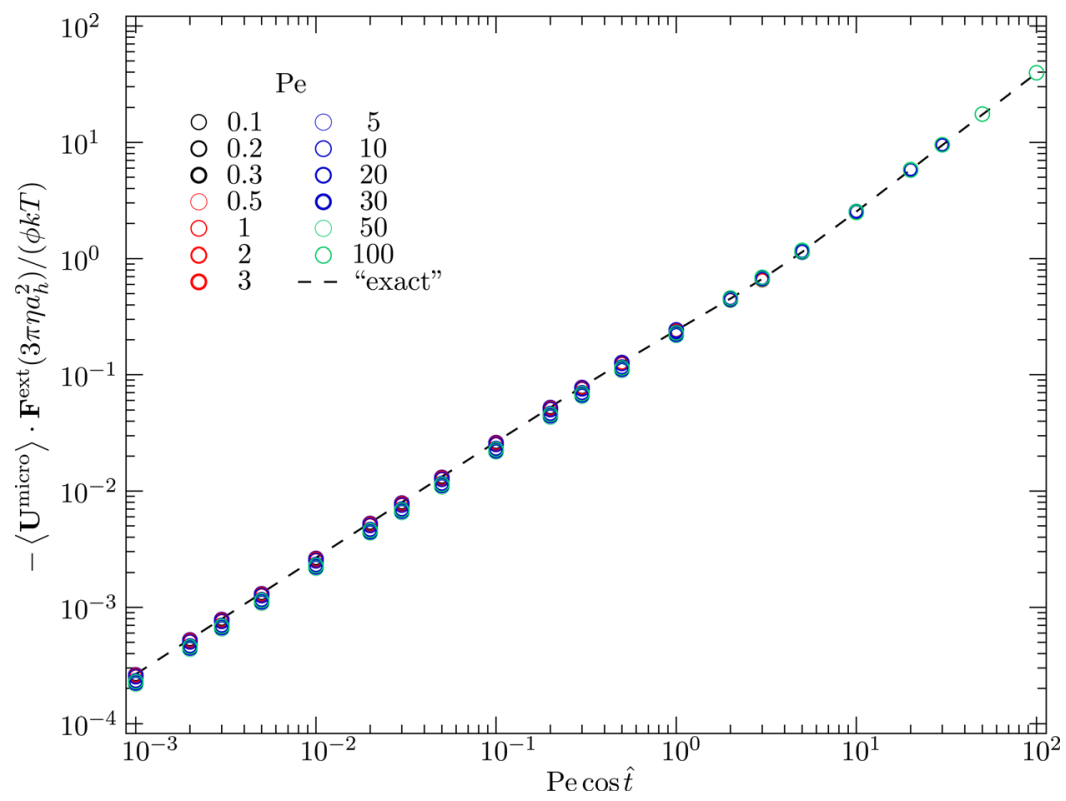

FIG. 6. The pseudosteady microvelocity, normalized by the diffusive velocity scale, $k T /\left(3 \pi \eta a_{h}^{2}\right)$, and the particle volume fraction, $\phi$, as a function of the pseudosteady Péclet number Pe $\cos \hat{t}$. The steady microvelocity normalized on this same scale and plotted as a function of the steady Péclet number, Pe, is indicated by the dashed line [Khair and Brady (2006)]. For a given maximum force amplitude, Pe, the pseudosteady microvelocity must recover the same value (i.e., the steady value) for all $|\mathrm{Pe} \cos \hat{t}|<\mathrm{Pe}$. This appears to be the case for all the values tested. 
pseudosteady response, the reduction in probe speed at these points should be identical for all values of Pe. As can be seen in Fig. 6, the data points are indistinguishable for $\mathrm{Pe} \cos \hat{t}>20$ and nearly so everywhere else. A deviation of less than $10 \%$ can be found for Pe $\cos \hat{t}<1$ with Pe $>20$, a predictable outcome of the truncation of the Fourier series at 20 terms. The Fourier mode $n=20$, for instance, allows the perturbed microstructure or the microvelocity to vary as $\cos (20 \hat{t})$ or $\sin (20 \hat{t})$ and to resolve temporal features on the dimensional time scale that is approximately $\omega^{-1} / 20$. The small deviation in the pseudosteady microvelocity from the steady result for Pe $>20$ with $\mathrm{Pe} \cos \hat{t}<1$ is the indistinguishability of temporal features on such a short time-scale. More Fourier modes would produce an even more accurate result, but as is plainly evident, the first twenty harmonics are sufficient for $\mathrm{Pe} \leq 100$.

We conclude by noting another interesting quality of the pseudosteady oscillation described by the low-frequency limit. In this regime, the Fourier modes of the microvelocity are all real. The first four odd harmonics are plotted in Fig. 7, where they are normalized by the value of the first mode when $\mathrm{Pe}=0$. The inset shows the first harmonic, and harmonics 3, 5, and 7 are given in the main plot. This reveals that the first harmonic encompasses more than $95 \%$ of the total signal in $\left\langle\mathbf{U}^{\text {micro }}\right\rangle$ at steady state. The third harmonic accounts for less than $5 \%$, and the fifth and seventh harmonics together comprise less than $2 \%$. The plot clearly shows that the first harmonic alone recovers force (shear) thinning and force (shear) thickening, two hallmark behaviors of hydrodynamically interacting colloidal dispersions. The sign of the ratio of modes $n=1$ and $n=3$ may indicate the intracycle shear-thinning and shear-thickening described by Ewoldt et al. (2008). However, the signature of steady-state processes and indeed the bulk of the viscous dissipation are almost entirely recovered in the first harmonic alone. Although this disparity in the values of the Fourier modes of the microvelocity allows one to understand the behavior via $n=1$ mode only, the solution of the governing equations for $f_{n}$ still requires determination of many coupled microstructural modes simultaneously.

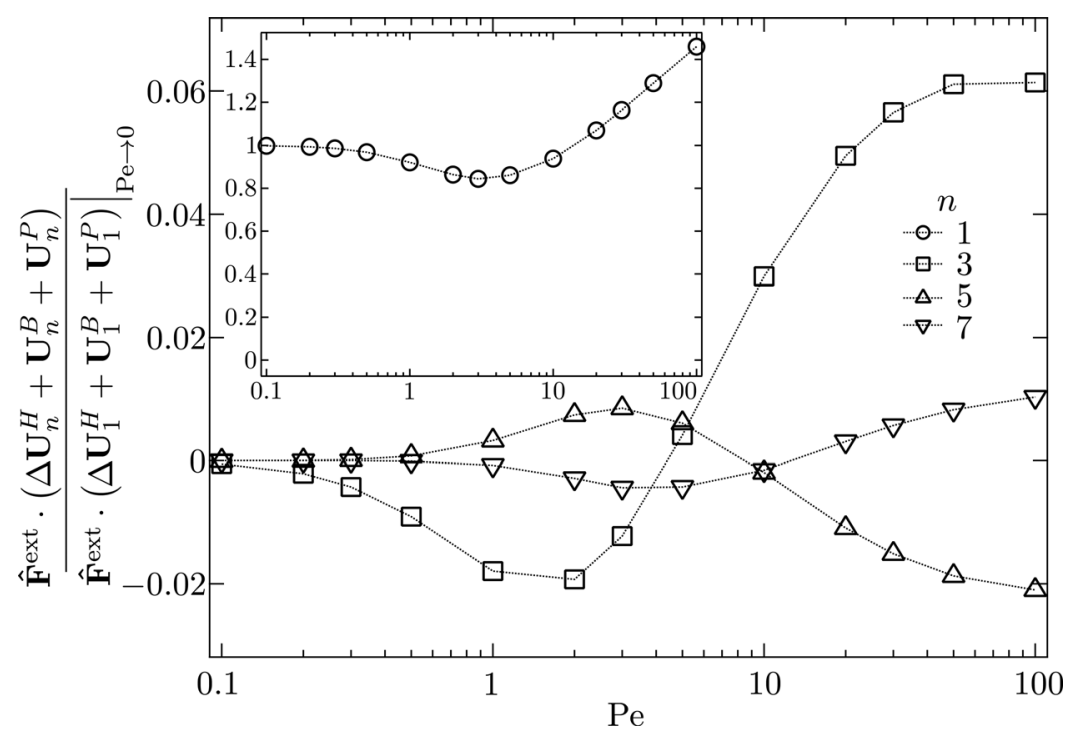

FIG. 7. Fourier modes of $\left\langle\mathbf{U}^{\text {micro }}\right\rangle$ plotted as a function of the Péclet number when $\alpha=0$. Each mode is normalized by the value of the first harmonic when $\mathrm{Pe}=0$. Main plot: Third, fifth, and seventh harmonics. Inset: First harmonic. The first harmonic comprises more than $95 \%$ of the total signal. 


\section{B. Arbitrary force amplitude and oscillation rate}

To study the entire range of forcing strength and oscillation rate depicted in Fig. 4, a numerical solution of the time dependent pair-Smoluchowski equation is required (cf. Sec. IV C 2). The oscillatory process is studied over a frequency range $\alpha \in[0,100]$ and a range of forces $\mathrm{Pe} \in[0,100]$. A parametric plot of input (external force) versus response (microvelocity) under a range of conditions is revealing. The time-dependent microvelocity of the probe (opposite the direction of the external force), $-\left\langle\mathbf{U}^{\text {micro }}\right\rangle \cdot \hat{\mathbf{F}}^{\text {ext }}$, is plotted versus the magnitude of the time-dependent external force, $F^{\operatorname{ext}} \cos (\omega t)$. These so-called Lissajous-Bowditch (L-B) curves are particularly helpful in understanding qualitative trends across a large parameter space. We begin with a brief review of the qualitative features of L-B curves and their application to microrheology.

\section{Interpretation of a Lissajous-Bowditch curve (L-B): Microrheology}

Figure 8 gives an illustration of several L-B curves. In this parametric plot, a straightline indicates a Newtonian microstructural response: The reduction in probe speed is exactly in-phase with the external force. In contrast, an ellipse with principle axes aligned horizontally and vertically indicates that the deformed microstructure acts as a Hookean solid: The slowing of the probe particle due to the microstructural deformation is $90^{\circ}$ out-of-phase with the external force. If the deformed microstructure behaves like a linear viscoelastic fluid, then the L-B curve is a tilted ellipse: The principle axes are no longer aligned horizontally and vertically. Finally, nonlinear viscoelasticity manifests as a Lissajous-Bowditch curve that is neither a line nor an ellipse. Placing all of the curves that result from an extensive parameter sweep in Pe and $\alpha$ on the same plot would quickly become difficult to read. However, one clever presentation of many related L-B curves is the Pipkin diagram.

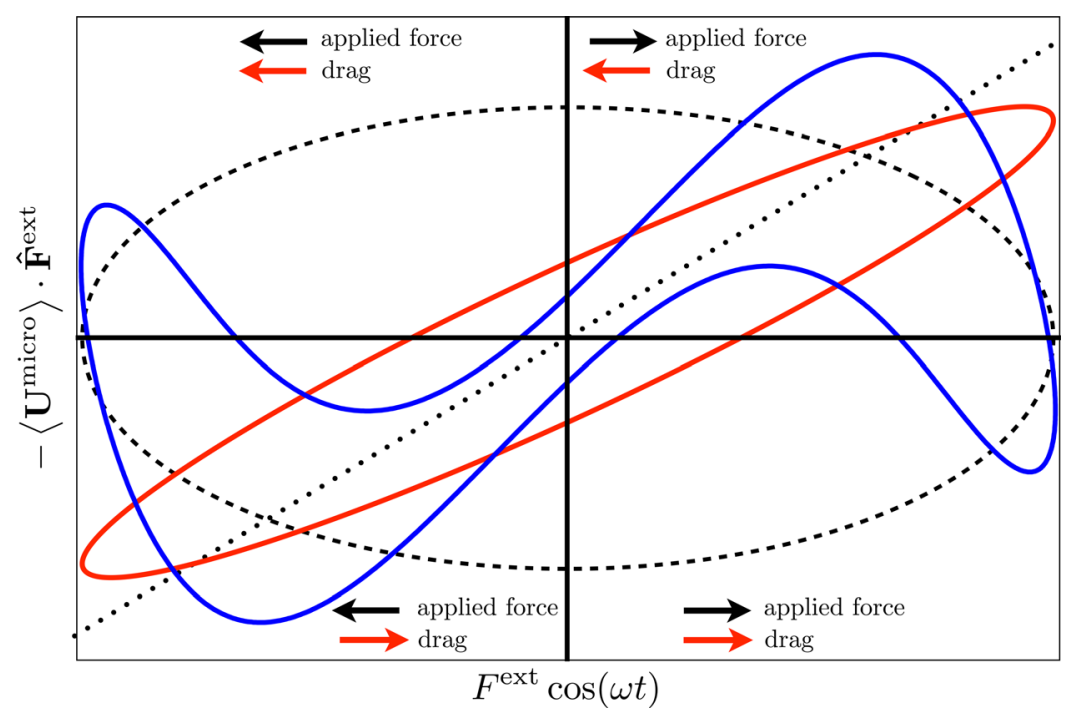

FIG. 8. Examples of Lissajous curves described by the microvelocity. The dotted line reflects how a microstructure acting as a Newtonian fluid would reduce the speed of the probe particle. The microvelocity is linear in and in phase with the oscillatory force. The dashed ellipse reflects how a microstructure acting as a Hookean solid would reduce the speed of the probe particle. The microvelocity is linear in and $90^{\circ}$ out of phase with the oscillatory force. The tilted ellipse is representative of a microstructure that acts as a linear viscoelastic fluid, while the nonelliptical curve is indicative of a nonlinear response. 


\section{A Pipkin diagram for microrheology}

A collection of Lissajous-Bowditch curves, termed a Pipkin diagram, is one means of comparing the time-dependent viscoelasticity exhibited under different flow conditions. We plot such a Pipkin diagram in Fig. 9, where the force and velocity axes have been normalized by their maximum value so that all the L-B curves associated with different force amplitudes and oscillation rates fill the space equally. For weak forces ( $\mathrm{Pe} \ll 1$ ), the L-B curves take on elliptical shapes for all frequencies. In fact, they appear independent of the strength of forcing for $\mathrm{Pe}<1$. This is the regime of linear viscoelasticity demarcated by $\mathrm{Pe} \ll 1$ or $\mathrm{Pe} / \alpha \ll 1$. Likewise, for slow oscillation $(\alpha \ll 1)$, the L-B curves contain practically no projected area. Thus, the response is viscous in nature. However, for stronger forcing, the deformed microstructure yields a non-Newtonian (thinning and then thickening) response-where downward concavity indicates thinning and upward concavity indicates thickening. This is the regime of steady nonlinear flow. There is a remaining region to consider where the external force is strong, $\mathrm{Pe}>1$, the oscillation is quick, $\alpha>1$, and the extent of deformation is not small, $\mathrm{Pe} / \alpha \approx 1$.

This regime lies in the bottom half of Fig. 9 near the diagonal. We refer to the response in this region as hypoviscous because the L-B curve is inverted, slanting from upper-left to lower-right. Such a slope indicates a negative increment to the viscosity due to the deformed microstructure. The microstructural conformations that lead to such a response are described in Sec. V C.

In Fig. 10, we plot the same Pipkin diagram, but first decompose the LissajousBowditch curves into their hydrodynamic and Brownian contributions. Again, each

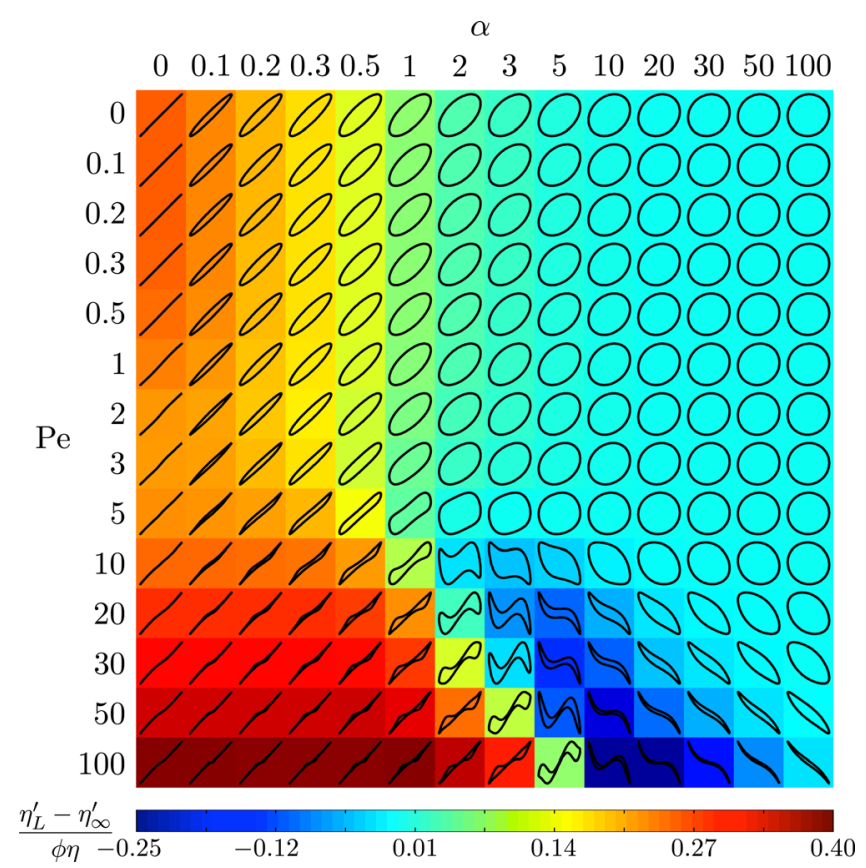

FIG. 9. Pipkin diagram: Lissajous curves for a range of forcing strengths $(\mathrm{Pe})$ and oscillation speeds $(\alpha)$. Each Lissajous curve (viscous projection) shows $-\left\langle\mathbf{U}^{\text {micro }}\right\rangle \cdot \hat{\mathbf{F}}^{\text {ext }}$ versus $F^{\mathrm{ext}} \cos (\alpha t)$ and is normalized to fill its subbox in the Pipkin diagram horizontally and vertically. The color behind each curves corresponds to the scale at the bottom of the figure and represents the slope of the line that connects the points of maximum and minimum force on the curve. We denote this $\eta_{L}^{\prime}-\eta_{\infty}^{\prime}$ following the notation of Ewoldt et al. (2008). This viscosity difference is normalized by the solvent viscosity and the particle volume fraction. 


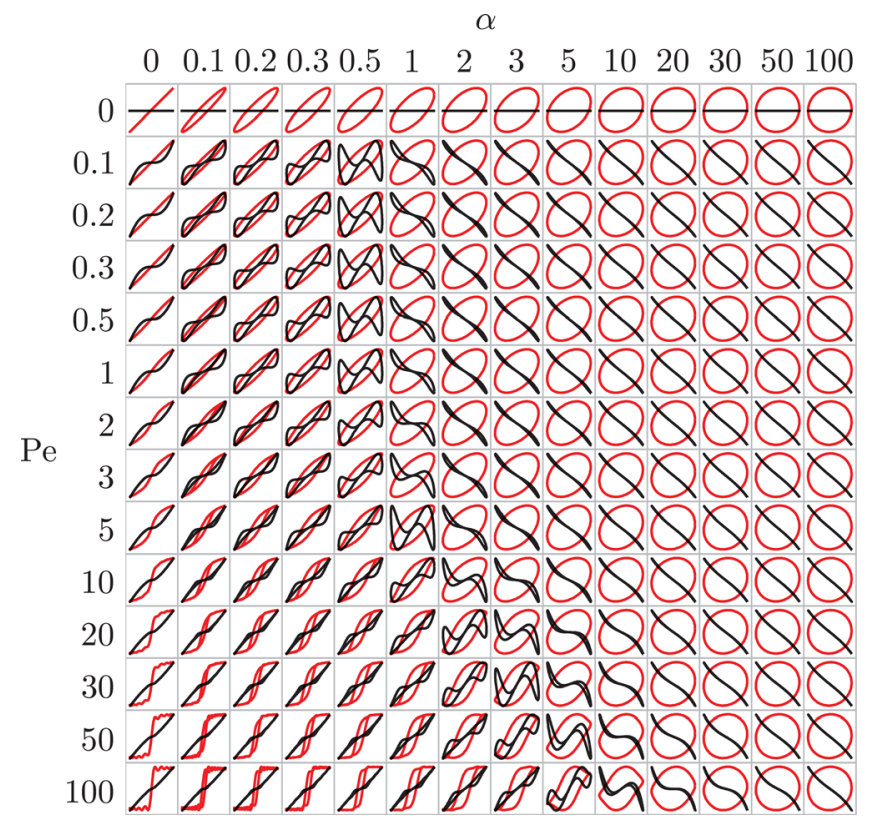

FIG. 10. Pipkin diagram (hydrodynamic and Brownian contributions shown separately): Lissajous curves from Fig. 9 have been separated here into hydrodynamic and Brownian contributions (black and grey (red online), respectively) to $-\left\langle\mathbf{U}^{\text {micro }}\right\rangle \cdot \hat{\mathbf{F}}^{\text {ext }}$ versus $F^{\text {ext }} \cos (\alpha t)$ and are normalized to fill their respective boxes horizontally and vertically.

response is scaled to fill the space equally. By comparison of curve shapes with Fig. 9, it is simple to determine which response is dominant. One qualitative difference between the hydrodynamic and Brownian contributions that stands out in such a diagram is the fact that the hydrodynamic response must pass through the origin. The hydrodynamic contribution to the microvelocity is at least linear in the time varying external force. Thus, when the no external force is applied, the hydrodynamic contribution is exactly zero. This is a signature of the purely dissipative nature of hydrodynamic forces. Over the course of a complete cycle, the hydrodynamic forces cannot lead to energy storage. The same is not true of the Brownian contributions, however. This important result shows that whenever a LissajousBowditch curve in Fig. 9 does not pass through the origin, the Brownian response is given explicitly by the value of the curve there. This is much the same as with cessation experiments performed in a macroscopic rheometer [Watanabe et al. (1996a, 1996b)]. While it can be quite difficult to extract the Brownian microstructural response by direct imaging of particles, in this approach one need only make observations of the Lissajous-Bowditch curves. Although the curves here are drawn from microrheology, this must also be true of macrorheology. Because the hydrodynamic stress result from macroscopic shear of a colloidal dispersion must be at least linear in the rate of strain, the points of zero shear rate on the Lissajous-Bowditch curve reflect nonhydrodynamic stresses only.

Given the intimate connection between energy dissipation and viscosity, it is natural to ask how much of the work done on the probe particle is dissipated per cycle

$$
Q=\frac{\alpha}{2 \pi} \int_{0}^{\frac{2 \pi}{\alpha}}\left\langle\mathbf{U}_{1}\right\rangle \cdot \mathbf{F}^{\mathrm{ext}} d t=Q^{\text {Stokes }}\left(1-U_{\infty}^{H} \phi\right)-Q^{\text {micro }},
$$

where $Q^{\text {Stokes }}=\left(F^{\text {ext }}\right)^{2} /\left(6 \pi \eta a_{h}\right)$ is the amount of energy dissipated per cycle in the absence of any bath particles, and 


$$
Q^{\text {micro }}=-\frac{\alpha}{2 \pi} \int_{0}^{\frac{2 \pi}{\alpha}}\left\langle\mathbf{U}^{\mathrm{micro}}\right\rangle \cdot \mathbf{F}^{\mathrm{ext}} d t
$$

is the change in the amount of energy dissipated per cycle by the deformed microstructure. Note that the $\left\langle\mathbf{U}^{\text {micro }}\right\rangle$ and $\mathbf{F}^{\text {ext }}$ are antiparallel in steady microrheology so that $Q^{\text {micro }}$ is positive. However, in fixed-force microrheology, the rate at which work done on the probe particle is dissipated is smaller than the Stokes result, $Q^{\text {Stokes }}$, precisely because the bath particles slow the probe down, hence seemingly superfluous but actually purposeful minus signs in Eqs. (57) and (58).

Because $\mathbf{F}^{\text {ext }}$ oscillates as a sinusoid with frequency $\alpha, Q^{\text {micro }}$ is proportional to the real part of the first harmonic of $\left\langle\mathbf{U}^{\text {micro }}\right\rangle$. Coming back to the connection between viscosity, velocity, and energy dissipation, we recognize in the ratio $Q^{\text {micro }} / Q^{\text {Stokes }}$ the real part of a complex viscosity. Let us define the increment to the complex viscosity, $\Delta \eta_{*}$, as the energy dissipated by (real) and stored in (imaginary) the deformed microstructure over a given cycle, $\Delta \eta_{*}=\Delta \eta_{*}^{\prime}-i \Delta \eta_{*}^{\prime \prime}$.

Thus, the viscosity increment $\Delta \eta_{*}^{\prime} / \eta=Q^{\text {micro }} / Q^{\text {Stokes }}$ describes how the cycleaveraged rate of energy dissipation is changed by the microstructural deformation. An alternative and more graphical method for quantifying the time dependent response was described by Ewoldt et al. (2008). Using their approach, one connects with a line the points of maximum and minimum force on each L-B curve (which occur at $t=0$ and $t=\pi / \alpha)$. The slope of this line characterizes a viscosity increment denoted $\Delta \eta_{L}^{\prime}$-see, for instance, the dotted line in Fig. 8 for a Newtonian fluid. One can easily show that $\Delta \eta_{L}^{\prime}$ is proportional to a sum over the real part of all the harmonic coefficients of $\left\langle\mathbf{U}^{\text {micro }}\right\rangle$. Thus, in the linear response limit, $\Delta \eta_{*}^{\prime} \equiv \Delta \eta_{L}^{\prime}$. But under nonlinear conditions, the two may differ. In the limit that $\alpha \rightarrow 0, \Delta \eta_{L}^{\prime}$ is equivalent to the steady microviscosity increment described by Squires and Brady (2005) and Khair and Brady (2006). As shown by Khair and Brady (2005), when $\alpha=0$ and $\mathrm{Pe}=0$, $\Delta \eta_{*}^{\prime}=\Delta \eta_{L}=0.262 \eta \phi$.

Both viscosity increments are plotted in Fig. 11. At low frequencies, the steady state response $(\alpha=0)$ dominates. Thus, the microstructural deformation produces an effect analogous to shear thinning and then thickening with increasing Péclet number. As the frequency increases and near $\mathrm{Pe}=0$, the viscosity increments become smaller exactly like the linear response solution. However, the viscosity increments still exhibit the same thinning followed by thickening with increasing Péclet number. The thinning is so pronounced that the viscosity increment actually becomes negative in one region. The total rate of energy dissipation is not negative, however. Recall that we have defined the microvelocity as the difference between the probe's average velocity and its high-frequency response. Thus, the total viscous dissipation per cycle due to the particle phase will be positive so long as $\Delta \eta_{*}^{\prime}>-U_{\infty}^{H} \phi=-1.828 \phi$. We term this state hypoviscous because, unexpectedly, less of the work done on the probe is dissipated by the deformed microstructure than by the microstructure where it undeformed (or equivalently, when deformed by an asymptotically high frequency oscillatory force). This seemingly puzzling result is easily understood if one recognizes that when the probe forcing is both strong and fast, it generates a wake depleted of bath particles which does not have time to relax and fill in before the probe returns to pass through it again - see the video provided in the supplementary material which reflects the conditions $\mathrm{Pe}=30, \alpha=3$.

There are an analogous set of elastic quantifiers of the Lissajous-Bowditch curves as well. Just as the viscous part of the complex viscosity $\Delta \eta_{*}^{\prime}$ is proportional to the real part of the first harmonic coefficient of $\left\langle\mathbf{U}^{\text {micro }}\right\rangle$, one may define 


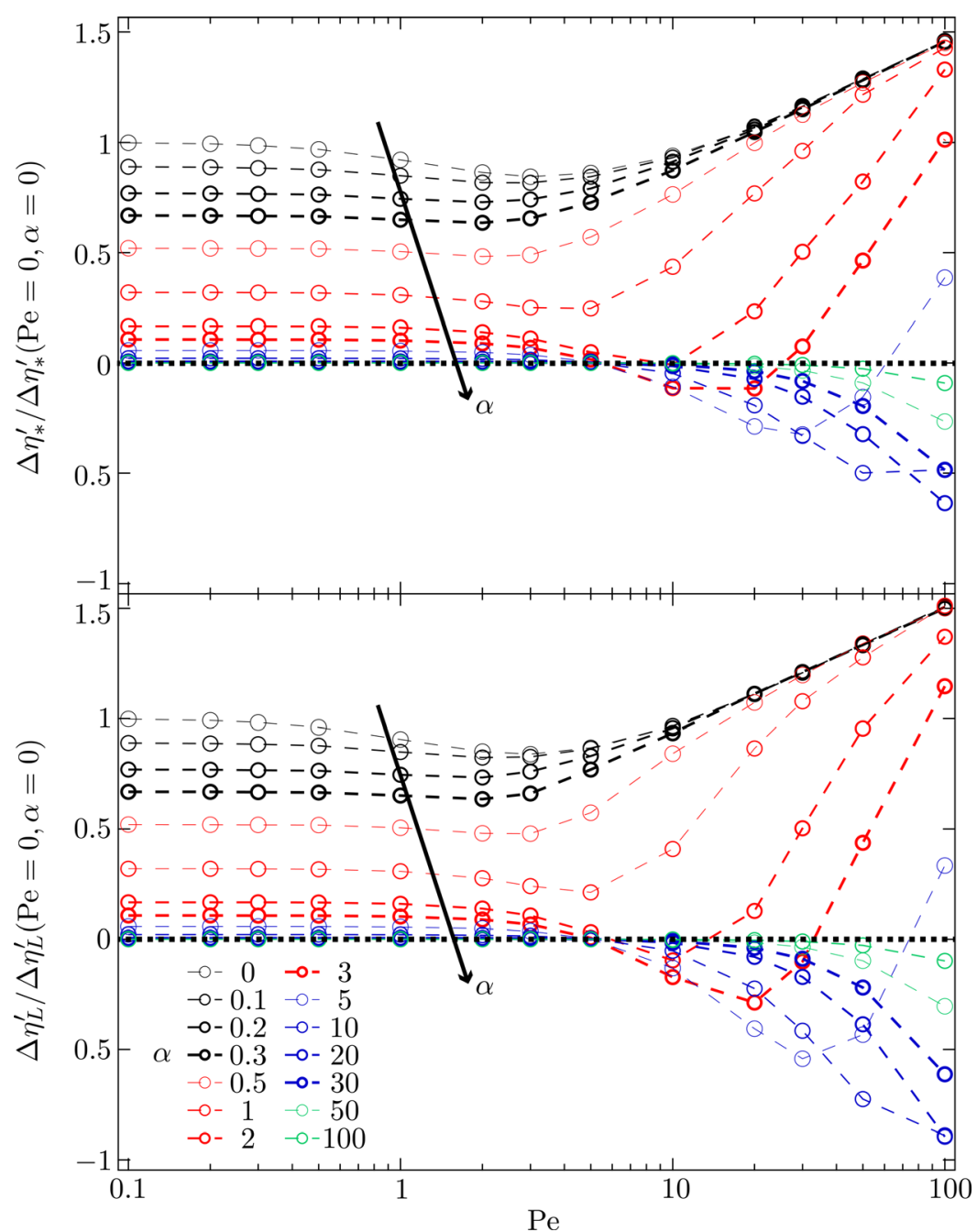

FIG. 11. The viscous part of the complex viscosity increments $\Delta \eta_{*}^{\prime}$ and $\Delta \eta_{L}^{\prime}$ plotted as a function of Pe and $\alpha$. The increments are identical in the linear-response limit but differ for Pe $>1$. Because $\Delta \eta_{L}^{\prime}$ comprises a sum over all harmonics of $\left\langle\mathbf{U}^{\text {micro }}\right\rangle$ (of which the first harmonic is the largest), differences between $\Delta \eta_{L}^{\prime}$ and $\Delta \eta_{*}^{\prime}$ arise from the higher $(n>1)$ response harmonics.

$$
\Delta \eta_{*}^{\prime \prime}=\frac{\alpha}{2 \pi}\left(\frac{6 \pi \eta a}{F^{\text {ext }}}\right) \int_{0}^{\frac{2 \pi}{\alpha}}\left\langle\mathbf{U}^{\text {micro }}\right\rangle \cdot \hat{\mathbf{F}}^{\text {ext }} \sin (\alpha t) d t,
$$

which extracts the imaginary part of the first harmonic coefficient of the microvelocity. This corresponds to the amount of elastic energy storage. In the linear response limit $(\mathrm{Pe} \rightarrow 0)$, $\Delta \eta_{*}$ is the complex viscosity increment described by Khair and Brady (2005). Likewise, elasticity can be interpreted graphically. Plotting $-\left\langle\mathbf{U}^{\text {micro }}\right\rangle \cdot \hat{\mathbf{F}}^{\text {ext }}$ versus $F^{\text {ext }} \sin (\alpha t)$ as a new L-B curve (not shown here), one can measure the slope of the line passing through the points of maximum and minimum impulse [proportional to $\left.F^{\mathrm{ext}} \sin (\alpha t)\right]$ at $t=\pi /(2 \alpha)$ and $t=3 \pi /(2 \alpha)$. This slope describes an elastic coefficient $\Delta \eta_{L}^{\prime \prime}$, whose viscous counterpart is $\Delta \eta_{L}^{\prime}$. The quantity $\Delta \eta_{L}^{\prime \prime}$ is given by a sum over the imaginary parts of the harmonic coefficients of $-\left\langle\mathbf{U}^{\mathrm{micro}}\right\rangle \cdot \mathbf{F}^{\mathrm{ext}}$ weighted by $(-1)^{n}$, where $n$ is the mode number. Thus, 
$\Delta \eta_{L}=\Delta \eta_{L}^{\prime}-i \Delta \eta_{L}^{\prime \prime}$ is another complex viscosity. Again, in the linear response limit, $\Delta \eta_{*}=\Delta \eta_{L}$, but these two diverge as the deformation becomes nonlinear. The quantity $\alpha \Delta \eta_{L}^{\prime \prime}$ is analogous to the elastic modulus $G_{L}$ described by Ewoldt et al. (2008).

The rate of deformation is a less relevant parameter with regard to elastic properties. Therefore, in Fig. 12, we plot the imaginary parts of the complex viscosities as a function of $\alpha$ for various values of Pe instead. From this we see that the elasticity grows and then decays with increasing frequency and for all Pe. The maximum in the elastic contribution shifts to higher frequency with increasing Peclet number. This suggests that the onset of the elastic plateau is delayed by the strength of the forcing. When we plot the frequency for which there is a peak in $\Delta \eta_{*}^{\prime \prime}$ or $\Delta \eta_{L}^{\prime \prime}$ as a function of Pe, we find that it scales as $\alpha \sim \mathrm{Pe}^{1 / 2}$ with $\mathrm{Pe} \gg 1$. We have not developed microstructural theory to predict this power law. However, the fact that the peak frequency is sublinear in the Peclet number is consistent with our prediction from Sec. V A 2 that the linear response, or equivalently the elastic plateau, dominates the probe motion when $\mathrm{Pe} / \alpha \ll 1$.

\section{Large-amplitude oscillations $(\alpha, \mathrm{Pe}, \mathrm{Pe} / \alpha \gg 1)$ : The hypoviscous regime}

So for, we have explored several regimes of the $(\mathrm{Pe}, \alpha)$ phase space via asymptotic and numerical analysis: Linear response, $\mathrm{Pe} \ll 1$; high frequency response

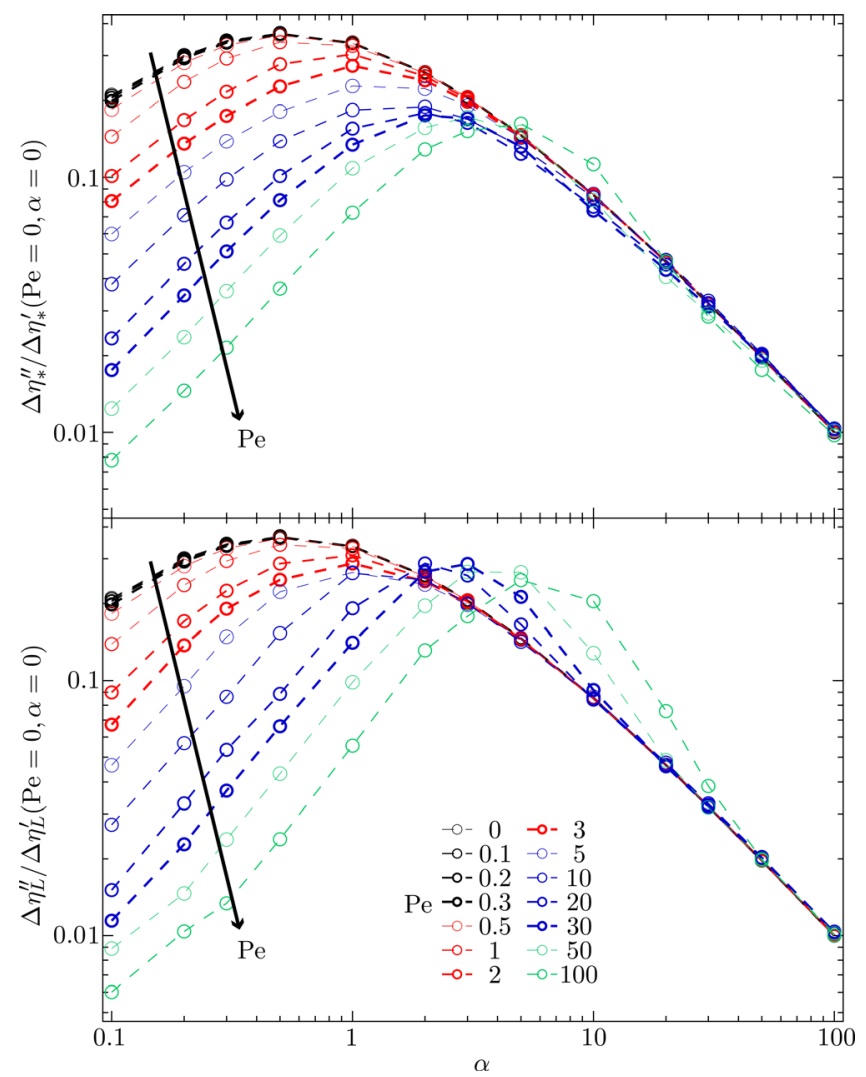

FIG. 12. The elastic part of the complex viscosity increment $\Delta \eta_{*}^{\prime \prime}$ and $\Delta \eta_{L}^{\prime \prime}$ as a function of Pe and $\alpha$. The increments are identical in the linear-response limit but differ for Pe $>1$. Because $\Delta \eta_{L}^{\prime}$ comprises a sum over all harmonics of $\left\langle\mathbf{U}^{\text {micro }}\right\rangle$ (of which the first harmonic is the largest), differences between $\Delta \eta_{L}^{\prime}$ and $\Delta \eta_{*}^{\prime}$ arise from the higher $(n>1)$ response harmonics. Linear-response behavior is recovered in the limit $\mathrm{Pe} / \alpha \ll 1$. In contrast to Fig. $11, \Delta \eta_{*}^{\prime \prime}$ and $\Delta \eta_{L}^{\prime \prime}$ are plotted against $\alpha$ for various values of Pe. 
$\alpha \gg 1, \operatorname{Pe} / \alpha \ll 1$; and steady response, $\alpha \ll 1$. There remains a single area of the phase space for which the forcing is strong, Pe $\gg 1$, the oscillation is rapid, $\alpha \gg 1$, and the extent of deformation is large, $\mathrm{Pe} / \alpha \gg 1$. Unlike the previous regimes, this one cannot be mapped directly onto well-known responses. This is the domain of large amplitude oscillatory forcing.

This regime reveals a unique viscoelastic state of the suspension. The hypoviscous state is characterized by a rate of viscous dissipation that is less than the corresponding rate in an undeformed suspension (dictated by the high frequency viscosity) per oscillatory cycle. A side-by-side examination of both the microstructural deformation and the microvelocity reveals the underlying cause of this behavior. The interplay between forcing strength and oscillation rate, and the large extent of deformation, $\Gamma=\mathrm{Pe} / \alpha$, is central to the physics producing the hypoviscous state.

Each of the two Lissajous-Bowditch curves plotted in Fig. 13 corresponds the microvelocity of a colloidal probe with $\Gamma=10$. However, Fig. 13(a) corresponds to $\mathrm{Pe}=3$, $\alpha=0.3$ while Fig. 13(b) corresponds to $\mathrm{Pe}=30, \alpha=3$. While the extent of deformation in both cases is large, $\mathrm{Pe} / \alpha=10$, they reside in quite different positions of the phase map (cf. Fig. 4). Figure 13(a) is closer to the linear response regime, while Fig. 13(b) is nearer the hypoviscous regime. The L-B curves show qualitatively very different behavior; to gain insight into the microstructural origins of these differences, a series of snapshots in time of the microstructure surrounding the probe accompanies each figure. The number in each snapshot matches the corresponding point on the L-B curve.

In case (a), we follow the L-B curve around counterclockwise from the point of maximum force (labeled 1) to the point of minimum force (labeled 5). There are pictures with corresponding labels for the microstructural deformation in which a positive external force drives the probe to the right. The arrows on the diagram indicate the direction and relative intensity of the microvelocity. Notably, the applied force and the microvelocity are antiparallel through much of the oscillatory cycle, indicating that there is little elasticity in the response. Moving from label 1 to label 3, the force on the probe particle is decreasing slowly with respect the microstructural relaxation rate $(\alpha<1)$, and in response the microstructural deformation decreases in intensity. However, because $\alpha$ is finite, the suspension retains some memory of its deformation when the external force is zero (label 3 ). This, of course, is the source of elasticity in the suspension. Now, from labels 3 to 5 , the external force on the probe particle grows more negative and the microstructural deformation grows more intense in response. The microstructural deformations labeled 1 and 5 are mirror images of each other, as they must be when symmetrically oscillating. This half of an oscillatory cycle is reversed when following the lower half of the L-B curve. Connecting points labeled 1 and 5 with a line we see that $\Delta \eta_{L}^{\prime}$ is positive.

For case (b), we begin again at label 1 and follow the L-B curve around counterclockwise. Label 1 corresponds to the maximum positive external force on the probe particle, yet unlike case (a), the microvelocity is parallel rather than antiparallel to the external force here. This puzzle is resolved by continuing to move around the L-B curve. At label 2 , the external force is still driving the probe particle to move to the right though it has decreased in magnitude. Note, the rate of oscillation of the external force is faster than the microstructural relaxation $(\alpha>1)$. Thus, the suspension does not relax quickly relative to the probe's motion, and the microstructural deformation grows more intense as further bath particles are caught in the boundary layer on the probe's leading edge. Likewise, the probe particle has created a substantial bath-particle-free wake which relaxes slow relative to the oscillation rate. At label 3, there is no longer an external force driving the probe particle, but microstructural deformation is still quite strong-especially when compared with case (a). When the force reverses direction, the probe particle 

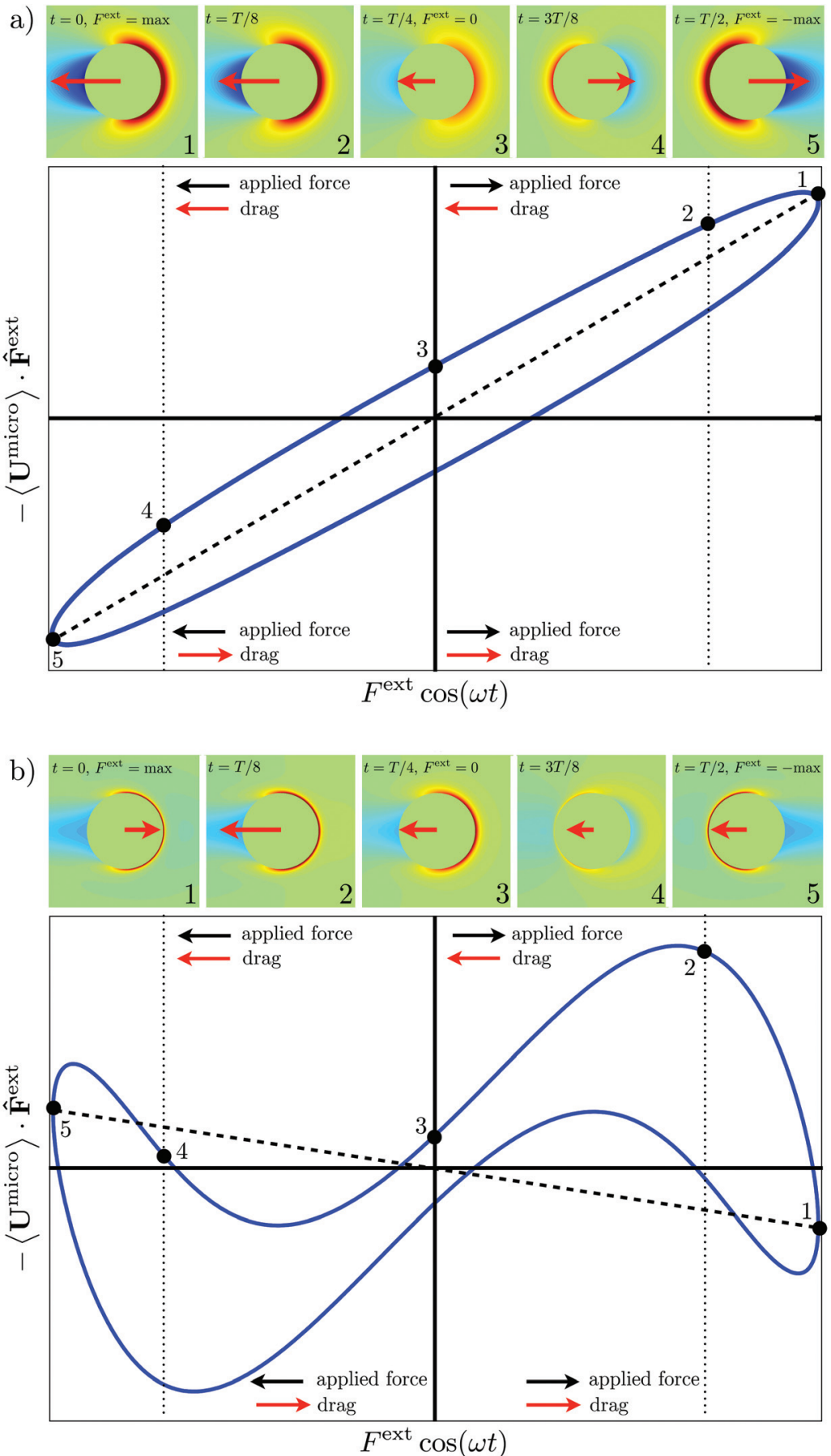

FIG. 13. Lissajous-Bowditch curves for large amplitude oscillatory microrheology under two conditions: (a) $\mathrm{Pe}=3, \alpha=0.3$ and (b) $\mathrm{Pe}=30, \alpha=3$. A panel of five contour plots of the microstructure surrounding the probe accompanies each figure. Each contour plot gives a snapshot in time of the oscillating structure, indicated by a number in the snapshot which matches the corresponding time in the Lissajous-Bowditch curve. The slope of the dashed line connecting points 1 and 5 indicate the sign of $\Delta \eta_{L}^{\prime}$. Videos corresponding to (a) and (b) are provided in the supplementary material. 
is driven into its own wake which has a low concentration of bath particles. At label 4, the deformed microstructure depicts explicitly the probe particle passing through the wake as the microstructure is depleted on the leading and trailing edges. Arriving at label 5 which is the mirror image of label 1 , we see now that the external force and microvelocity are parallel because the probe particle has not finished moving through the wake. The probe must continue traveling to the left until it reaches the boundary of the wake and begins accumulating bath particles on its leading edge. Connecting points labeled 1 and 5 with a line we see that $\Delta \eta_{L}^{\prime}$ is negative.

Recall that the reduction in probe speed due to hindrance of the bath is divided into two contributions: The first is a purely hydrodynamic contribution arising from flow of solvent between the bath particles given their equilibrium configuration, $-U_{\infty}^{H} \phi$; the second arises due to hydrodynamic and conservative interactions propagated through the deformed microstructure. Thus if the deformed microstructure near the probe is depleted of bath particles [i.e., $f(\mathbf{r}, t)<0$, for $r \approx 2$ ], the local deformation should actually enable the probe to move faster, rather than hindering its motion. This is indeed what is observed. It is import to note, however, that the hypoviscous effect is always accompanied by the condition $-\mathbf{U}^{\text {micro }} \cdot \hat{\mathbf{F}}^{\text {ext }}<U_{\infty}^{H} \phi$; that is, the particle phase as a whole hinders probe motion.

The hypoviscous effect is one that may be unique to large amplitude oscillatory deformations since it reflects memory of the nonlinear microstructural deformation. A similar effect was observed in the eighties during the study of large amplitude oscillatory shear of non-Brownian suspensions where the thermal relaxation of the particles is vanishingly slow. The analogous dimensionless groups diverge: $\mathrm{Pe} \rightarrow \infty$ and $\alpha \rightarrow \infty$; however, their ratio is set by the extent of deformation - the strain amplitude. In their study of shearinduced structure in non-Brownian suspensions, Gadala-Maria and Acrivos (1980) conducted flow-reversal experiments on suspensions of hard spheres. When a "fresh" suspension undergoes shear, there is a transient regime in which the viscosity climbs to its steady-state value. However, they found that upon stopping the flow (dropping the stress to zero) and then restarting it, the viscosity jumped instantaneously to its steady-state value with no transient period. However, upon restarting the flow in the opposite direction, the viscosity decreased and then gradually recovered its steady value. No wait period between flow cessation and reversal changed these results. From these observations, they concluded in part that the flow deforms the arrangement of particles, and that this structure would "remember" the deformation for an indefinite period of time. Without thermal motion of the particles, the low-Reynolds-number hydrodynamic interactions are perfectly reversible. Thus, the equilibrium structure is nearly always recovered at zero strain. The thermal motion in a Brownian suspension stymies this reversibility and leads to memory of the deformation history. The hypoviscous effect appears robust and stems from the break in symmetry between relaxation of compressed and extended regions of the material microstructure.

\section{Interpretation of large amplitude oscillatory deformation experiments}

Many approaches have been proposed for inferring material properties and flow behavior from large-amplitude oscillatory deformation. Some arguments put forth include geometric interpretations of Lissajous-Bowditch curves or physical interpretations based on constitutive models. However, these suffer from the fact that in the nonlinear regime, such interpretations are not deformation-agnostic. Unlike linear response, the details of the deformation and deformation history are inextricably coupled. That is, there is likely no simple transformation connecting the shear stress under one nonlinear 
time-dependent flow to that arising in another. From such a perspective, a suite of largeamplitude oscillatory experiments sweeping through a range of amplitude and frequency are difficult to connect by a common material modulus.

An alternative is to accept the limitations of the large amplitude oscillation experiment and ask what physical information can be unambiguously extracted. One such measure is the amount of energy a material dissipates in response to deformation. The rate of energy dissipation in a material with shear stress $\sigma(t)$ and strain rate tensor $\dot{\gamma}(t)$ is

$$
\dot{Q}=\sigma(t) \dot{\gamma}(t)
$$

The average rate of work done on the material that is dissipated as heat in the time interval $\left[t_{0}, t_{0}+T\right]$ is

$$
\overline{\dot{Q}}=\frac{1}{T} \int_{t_{0}}^{t_{0}+T} \sigma(t) \dot{\gamma}(t) d t .
$$

In a large amplitude oscillatory shear experiment, the rate of strain is $\dot{\gamma}(t)=\dot{\gamma}_{0} \cos (\omega t)$ and the shear stress can be written as $\sigma(t)=\dot{\gamma}_{0} \sum_{n} \exp (i n \omega t) A_{n}$ where the indices of the summation are odd and run between $\pm \infty$. If the length of the interval is a single strain cycle, $T=2 \pi / \omega$, then the average rate of work dissipated as heat is

$$
\overline{\dot{Q}}=\dot{\gamma}^{2} \mathcal{R}\left\{A_{1}\right\} .
$$

The simplest possible measurement made from a LAOS experiment also provides the most physical characterization. The real part of the first harmonic of the shear stress reflects the average rate of energy dissipation per cycle. Thus, this part of the coefficient may be termed "viscous" or "dissipative" unambiguously. Naturally, $\mathcal{R}\left\{A_{1}\right\}$ has units of viscosity. If the interval over which the average rate of energy dissipation is measured is half a cycle so that $T=\pi / \omega, \dot{Q}$ is the same as over the full cycle. This reflects the odd symmetry of the shear stress with respect to the rate of strain. The average rate of energy dissipated over any interval that is an integer fraction, $m$, of the period, $T=2 \pi /(m \omega)$, is

$$
\overline{\dot{Q}}_{m}\left(t_{0}\right)=\dot{\gamma}^{2} \mathcal{R}\left\{A_{1}\right\}+\frac{m \omega}{2 \pi} \frac{\dot{\gamma}^{2}}{2} \sum_{n \in \text { even } \neq 0} e^{i n \omega t_{0}}\left(A_{n-1}+A_{n+1}\right) \frac{e^{2 \pi i n / m}-1}{i n \omega}
$$

and depends on when during the cycle the measurement of dissipation is made. Thus, both the real and imaginary parts of the higher order harmonics can be thought of as contributing to the viscous dissipation during portions of the oscillation and cannot be uniquely tied to elastic processes, for instance.

In a similar sense, the elastic energy stored in a deformed material can be defined in terms of the product of shear stress and strain,

$$
E=\sigma(t) \gamma(t)
$$

where $\gamma(t)=\gamma_{0} \sin (\omega t)$. As with the average dissipation, an average amount of energy stored per cycle is

$$
\bar{E}=\frac{\omega}{2 \pi} \int_{t_{0}}^{t_{0}+2 \pi / \omega} \sigma(t) \gamma(t) d t=-\frac{\dot{\gamma}_{0}^{2}}{\omega} \mathcal{I}\left\{A_{1}\right\}
$$


Therefore, the imaginary part of the first harmonic of the stress in response to a large amplitude oscillatory deformation can be uniquely identified with the cycle averaged amount of elastic storage. This may be termed unambiguously conservative or "elastic." A similar construction for the average amount of elastic energy stored over some fractional portion of a cycle will indicate that the higher order harmonics are fundamentally viscoelastic and cannot be used to describe viscous dissipation or elastic storage uniquely during any finite portion of the oscillation.

We have used a microrheological analog to these measures to show that the most interesting features arising from a large amplitude oscillatory deformation are apparent in the first harmonics of the response. It seems likely that the first harmonic is the only necessary quantitative descriptor of the deformation process and that this in conjunction with a sweep of frequency and amplitude should be sufficient for parameter estimation in a constitutive model. Given that the higher harmonics can be orders of magnitude smaller than first harmonic, this also seems a sound method for practical application of large amplitude oscillatory deformation data.

\section{E. A brief note on scaling results for higher concentrations}

We have been careful throughout to examine the time varying velocity of the probe in a fashion that removes the deformation independent, high frequency response. The growth of this deformation independent part with increasing concentration is known to coincide with the short-time self-diffusivity of a colloidal particle in a suspension [Khair and Brady (2005)]. Thus, this contribution can be handled trivially. The deformation dependent part of the response has been shown to scale in concentration with the difference between the long- and short-time self-diffusivities: $D_{\infty}^{S}(\phi)-D_{0}^{S}(\phi)$. Thus, a scaled-up prediction of the probe velocity would be

$$
\mathbf{U}_{1}=\frac{D_{0}^{S}(\phi)}{k T} \cos (\omega t) \mathbf{F}^{\mathrm{ext}}-\frac{D_{\infty}^{S}(\phi)-D_{0}^{S}(\phi)}{0.27 \phi}\left\langle\mathbf{U}^{\mathrm{micro}}\right\rangle,
$$

where $\left\langle\mathbf{U}^{\text {micro }}\right\rangle$ refers to the dilute limit microvelocity increment derived in this article, and $-0.27 \phi$ is $D_{\infty}^{S}(\phi)-D_{0}^{S}(\phi)$ expressed to the same order in $\phi$ as the dilute theory.

One additional complication associated with scaling up is the change in particle relaxation kinetics in more concentrated dispersions. Thus to compare the dilute theory to experiments, it is necessary to establish an equivalent dimensionless force, Péclet number, and dimensionless oscillation frequency, $\alpha$. In past work, it was determined that the slower short-time self-diffusion of particles in concentrated suspensions accounts for this effect [Khair and Brady (2006)]. Thus, we can replace the magnitude of the diffusivity, $D$, in our earlier attempts to render to the Smoluchowski equation dimensionless with the relative short-time self-diffusivity between probe and bath particles: $2 D_{0}^{S}(\phi)$. We define the Péclet number in concentrated dispersions as $\mathrm{Pe}=F^{\mathrm{ext}} /\left(12 \pi \eta D_{0}^{S}(\phi)\right)$. Similarly, we prescribe $\alpha=\omega a_{h}^{2} /\left(2 D_{0}^{S}(\phi)\right)$. This will allow for a proper basis of comparison between the dilute theory and experiments.

\section{CONCLUSION}

A microstructural perspective on large amplitude oscillatory deformation is useful for understanding the relative importance of different forces, conservative and nonconservative, in determining a material's unsteady and nonlinear flow properties. We have studied a colloidal dispersion deformed by a probe particle driven with an oscillatory force of 
arbitrary magnitude and frequency. This is the microrheological analogue to the LAOS experiment. The response of the suspension to this deformation falls into four regimes which are characterized by the relative magnitudes of the force amplitude, represented by $\mathrm{Pe}=F^{\mathrm{ext}} a /(2 k T)$, and the oscillation rate, represented by $\alpha=6 \pi \eta a_{h} a^{2} \omega / k T$. An additional relevant parameter is given by the ratio of these two quantities, $\mathrm{Pe} / \alpha$, the extent of deformation which is analogous to the strain amplitude in a LAOS experiment.

When $\mathrm{Pe} \ll 1$, the force on the probe particle is weak and the response of the suspension to deformation is given by its linear viscoelastic spectrum. When $\alpha \gg 1$ and $\mathrm{Pe} / \alpha \ll 1$, the oscillation is quick while the extent of deformation in the suspension is small - the linear response is also recovered. When $\mathrm{Pe} \gg 1$ and $\alpha \ll 1$, the force is strong while the oscillation is slow-the response of the suspension is pseudosteady and nonlinear. Finally, when $\mathrm{Pe} \gg 1, \alpha \gg 1$, and $\mathrm{Pe} / \alpha \gg 1$, the force on the probe is strong, the oscillation is quick and the extent of deformation is large. In this last regime, we discovered the hypoviscous state in which oscillations lead to a locally depleted microstructure and a reduction from equilibrium in the averaged hydrodynamic resistance to deformation. In another material with a single relaxation time scale, these same four regimes delineated by extreme values of $\alpha, \mathrm{Pe}$, and $\mathrm{Pe} / \alpha$ likely persist, though the response within these regimes may differ. The hypoviscous effect, for instance, is a purely hydrodynamic product, so in a material with weak hydrodynamic stresses, this may not be observed. Nonetheless, simple dimensional analysis and a microstructural view of the deformed material offer great insight into nonlinear viscoelasticity.

The approach taken in this article is prototypical of microstructural analysis of complex materials. It is by no means limited to the realms of microrheology and colloidal dispersions - though those both provide many convenient analytical footholds. It seems worthwhile to interrogate the microstructural response and the stresses that result from the oscillatory driving of other materials and subject to other modes of deformation. One obvious extension of this work is the investigation of suspensions of hard-spheres deformed by an oscillatory, simple shear. Similar analyses must be possible for other microstructured materials such as polymer solutions and melts. These would provide a valuable theoretical framework, beyond constitutive modeling, for the interpretation of LAOS experiments which have become all but de rigeur in recent years.

\section{ACKNOWLEDGMENTS}

The authors thank Professors Eric Furst, Norman, Wagner and Itai Cohen for helpful discussions about LAOS. Support from NASA is gratefully acknowledged by JWS (Grant Nos. NAG3-2832 and NNX07AD02G).

\section{APPENDIX: ANALYSIS OF LAOS FROM THE N-PARTICLE PERSPECTIVE}

Consider a suspension of $N$ spherical particles. The flux of particle $\alpha$ is denoted

$$
\mathbf{j}_{\alpha}\left(\mathbf{x}^{N}, t\right)=\left[\mathbf{U}_{\alpha}\left(\mathbf{x}^{N}, t\right)-\sum_{\beta=1}^{N} \mathbf{D}_{\alpha \beta}\left(\mathbf{x}^{N}\right) \cdot \nabla_{\beta}\left(\frac{V\left(\mathbf{x}^{N}\right)}{k T}+\log P_{N}\left(\mathbf{x}^{N}, t\right)\right)\right] P_{N}\left(\mathbf{x}^{N}, t\right),
$$

where $\mathbf{x}^{N}$ is a vector representing the positions of all the particle centers and $P_{N}\left(\mathbf{x}^{N}, t\right)$ is the probability of finding them in that configuration at time $t$. At equilibrium, $P_{N}\left(\mathbf{x}^{N}, t\right)=P_{N}^{\mathrm{eq}}\left(\mathbf{x}^{N}\right) \sim \exp \left[-V\left(\mathbf{x}^{N}\right) / k T\right]$, where $V\left(\mathbf{x}^{N}\right)$ is the conservative potential among the particles. Then, $\mathbf{U}_{\alpha}\left(\mathbf{x}^{N}, t\right)$ is the velocity of particle $\alpha$ due to nonconservative forces. 
For the present purposes, it will suffice to assume that it is independent of the particle probability distribution. We write this probability distribution as a perturbation about its equilibrium value so that $P_{N}\left(\mathbf{x}^{N}, t\right)=P_{N}^{\mathrm{eq}}\left(\mathbf{x}^{N}\right)\left[1+f_{N}\left(\mathbf{x}^{N}, t\right)\right]$. The flux of particle $\alpha$ is then

$$
\mathbf{j}_{\alpha}\left(\mathbf{x}^{N}, t\right)=P_{N}^{\mathrm{eq}}\left(\mathbf{x}^{N}\right)\left[\mathbf{U}_{\alpha}\left(\mathbf{x}^{N}, t\right)\left[1+f_{N}\left(\mathbf{x}^{N}, t\right)\right]-\sum_{\beta=1}^{N} \mathbf{D}_{\alpha \beta}\left(\mathbf{x}^{N}\right) \cdot \nabla_{\beta} f_{N}\left(\mathbf{x}^{N}, t\right)\right] .
$$

If we assume that $\mathbf{U}_{\alpha}\left(\mathbf{x}^{N}, t\right)$ can be decomposed into $\tilde{\mathbf{U}}_{\alpha}\left(\mathbf{x}^{N}\right) \cos (\omega t)$ as would be the case for a dispersion driven by an oscillatory flow or force, then the microstructural perturbation can be expanded as

$$
f_{N}\left(\mathbf{x}^{N}, t\right)=\sum_{n=-\infty}^{\infty} e^{i n \omega t} f_{N}^{(n)}\left(\mathbf{x}^{N}\right)
$$

where the $f_{N}^{(n)}\left(\mathbf{x}^{N}\right)$ are the microstructural harmonics. The flux of particle $\alpha$ can be expanded likewise such that

$$
\mathbf{j}_{\alpha}=\sum_{n=-\infty}^{\infty} e^{i n \omega t} \mathbf{j}_{\alpha}^{(n)}\left(\mathbf{x}^{N}\right)
$$

where $\mathbf{j}_{\alpha}^{(n)}\left(\mathbf{x}^{N}\right)$ is

$$
P_{N}^{\mathrm{eq}}\left(\mathbf{x}^{N}\right)\left\{\frac{1}{2} \tilde{\mathbf{U}}_{\alpha}\left(\mathbf{x}^{N}\right)\left[\delta_{1 n}+\delta_{-1 n}+f_{N}^{(n-1)}\left(\mathbf{x}^{N}\right)+f_{N}^{(n+1)}\left(\mathbf{x}^{N}\right)\right]-\sum_{\beta=1}^{N} \mathbf{D}_{\alpha \beta}\left(\mathbf{x}^{N}\right) \cdot \nabla_{\beta} f_{N}^{(n)}\left(\mathbf{x}^{N}\right)\right\} .
$$

The $N$-particle Smoluchowski equation states

$$
\dot{P}_{N}\left(\mathbf{x}^{N}, t\right)=-\sum_{\alpha=1}^{N} \nabla_{\alpha} \cdot \mathbf{j}_{\alpha}
$$

where upon matching orthogonal modes,

$$
\operatorname{in\omega } P_{N}^{\mathrm{eq}}\left(\mathrm{x}^{N}\right) f_{N}^{(n)}\left(\mathbf{x}^{N}\right)=-\sum_{\alpha=1}^{N} \nabla_{\alpha} \cdot \mathbf{j}_{\alpha}^{(n)}\left(\mathbf{x}^{N}\right),
$$

for $n=0, \pm 1, \pm 2, \ldots$. The structure of this equation is obviously the same as Eq. (15). Thus, the analysis of the microstructure presented in the article can be easily extended to the $N$-particle perspective while allowing incorporation of other interparticle potentials and flow profiles.

\section{References}

App, E. M., R. Kieselmann, D. Reinhardt, H. Lindemann, B. Dasgupta, M. King, and P. Brand, "Sputum rheology changes in cystic fibrosis lung disease following two different types of physiotherapy: Flutter vs autogenic drainage," Chest 114, 171-177 (1998).

Banerjee, R., J. Bellare, and R. Puniyani, "Effect of phospholipid mixtures and surfactant formulations on rheology of polymeric gels, simulating mucus, at shear rates experienced in the tracheobronchial tree," Biochem Eng J. 7, 195-200 (2001). 
Barnes, H. A., "Shear-thickening (dilatancy) in suspensions of nonaggregating solid particles dispersed in Newtonian liquids," J. Rheol. 33, 329-366 (1989).

Barnes, H. A., F. F. Hutton, and K. Walters, An Introduction to Rheology (Elsevier, Amsterdam, 1989).

Batchelor, G. K., "Sedimentation in a dilute dispersion of spheres," J. Fluid Mech. 52(2), 245-268 (1972).

Batchelor, G. K., "Brownian diffusion of particles with hydrodynamic interaction," J. Fluid Mech. 74, 1-29 (1976).

Batchelor, G. K., and J. T. Green, “The hydrodynamic interaction of two small freely-moving spheres in a linear flow field,” J. Fluid Mech. 56(2), 375-400 (1972).

Bender, J. W., and N. J. Wagner, "Optical measurement of the contributions of colloidal forces to the rheology of concentrated suspensions,” J. Colloid Interface Sci. 172, 171-184 (1995).

Bergenholtz, J., J. F. Brady, and M. Vicic, "The non-Newtonian rheology of dilute colloidal suspensions," J. Fluid Mech. 456, 239-275 (2002).

Brady, J. F., "Brownian motion, hydrodynamics, and the osmotic pressure," J. Chem. Phys. 98, 3335-3341 (1993a).

Brady, J. F., "The rheological behavior of concentrated colloidal dispersions," J. Chem. Phys. 99, 567-581 (1993b).

Brady, J. F., and G. Bossis, "The rheology of concentrated suspensions of spheres in simple shear flow by numerical simulation,” J. Fluid Mech. 155, 105-129 (1985).

Breedveld, V., and D. J. Pine, "Microrheology as a tool for high-throughput screening," J. Mater. Sci. 38, 4461-4470 (2003).

Cheng, X., J. H. McCoy, J. N. Israelachvili, and I. Cohen, "Imaging the microscopic structure of shear thinning and thickening colloidal suspensions," Science 333(6047), 1276-1279 (2011).

Cho, K. S., K. H. Ahn, and S. J. Lee, "A geometrical interpretation of large amplitude oscillatory shear response,” J. Rheol. 49, 747-758 (2005).

Crocker, J. C., "Measurement of the hydrodynamic corrections to the Brownian motion of two colloidal spheres," J. Chem. Phys. 106, 2837-2840 (1997).

Crocker, J. C., and D. G. Grier, "Methods of digital video microscopy for colloidal studies," J. Colloid Interface Sci. 179, 298-310 (1996).

Crocker, J. C., M. T. Valentine, E. R. Weeks, T. Gisler, P. D. Kaplan, A. G. Yodh, and D. A. Weitz, “Two-point microrheology of inhomogeneous soft materials," Phys. Rev. Lett. 85(4), 888-891 (2000).

Dealy, J. M., and K. F. Wissbrun, Melt Rheology and Its Role in Plastics Processing: Theory and Applications (Van Nostrand, Reinhold, New York, 1990).

D'Haene, P., J. Mewis, and G. G. Fuller, "Scattering dichroism measurements of flow-induced structure of a shear thickening suspension,” J. Colloid Interface Sci. 156, 350-358 (1993).

Einstein, A., "On the theory of the Brownian movement," Ann. Phys. 19, 371-381 (1906).

Ewoldt, R. H., A. Hosoi, and G. H. McKinley, "New measures for characterizing nonlinear viscoelasticity in large amplitude oscillatory shear," J. Rheol. 52, 1427-1458 (2008).

Foss, D. R., "Rheological behavior of colloidal suspensions: The effects of hydrodynamic interactions," Ph.D. thesis, California Institute of Technology, 1999.

Freundlich, H., and W. Seifriz, "Über die elästizitaet von solen und gelen," Z. Phys. Chem. 104, 233-261 (1923).

Gadala-Maria, F., and A. Acrivos, "Shear-induced structure in a concentrated suspension of solid spheres," J. Rheol. 24(6), 799-814 (1980).

Gao, C., S. D. Kulkarni, J. F. Morris, and J. F. Gilchrist, "Direct investigation of anisotropic suspension structure in pressure-driven flow," Phys. Rev. E. 81, 041403 (2010).

Giacomin, A. J., and J. M. Dealy, "Large-amplitude oscillatory shear," in Techniques in Rheological Measurement (Elsevier Applied Science, London, 1993), Chap. 4.

Gisler, T., and D. A. Weitz, "Scaling of the microrheology of semidilute f-actin solutions," Phys. Rev. Lett. 82, 1606-1610 (1999).

Gurnon, K. A., and N. J. Wagner, "Large amplitude oscillatory shear (laos) measurements to obtain constitutive equation model parameters: Giesekus model of banding and nonbanding wormlike micelles," J. Rheol. 56(2), 333-352 (2012). 
Habdas, P., D. Schaar, A. C. Levitt, and E. R. Weeks, "Forced motion of a probe particle near the colloidal glass transition," Europhys. Lett. 67, 477-483 (2004).

Hyun, K., S. Kim, K. Ahn, and S. Lee, "Large amplitude oscillatory shear as a way to classify the complex fluids,” J. Non-Newtonian Fluids 107(1-3), 51-65 (2002).

Jeffrey, D. J., and Y. Onishi, "Calculation of the resistance and mobility functions for two unequal rigid spheres in low-Reynolds-number flow,” J. Fluid Mech. 139, 261-290 (1984).

Kaffashi, B., V. T. O'Brien, M. E. Mackay, and S. M. Underwood, "Elastic-like and viscous-like components of the shear viscosity for nearly hard sphere, Brownian suspensions," J. Colloid Interface Sci. 187, 22-28 (1997).

Khair, A. S., and J. F. Brady, “'Microviscoelasticity’ of colloidal dispersions,” J. Rheol. 49, 1449-1481 (2005).

Khair, A. S., and J. F. Brady, "Single particle motion in colloidal dispersions: A simple model for active and nonlinear microrheology," J. Fluid Mech. 557, 73-117 (2006).

Kim, S., and S. J. Karrila, Microhydrodynamics: Principles and Selected Applications (ButterworthHeinemann, Boston, 1991).

Lai, S. K., Y.-Y. Wang, D. Wirtz, and J. Hanes, "Micro- and macrorheology of mucus," Adv. Drug Delivery Rev. 61(2), 86-100 (2009).

Levine, A. J., and T. C. Lubensky, "One- and two-particle microrheology," Phys. Rev. Lett. 85, 1774-1778 (2000).

Lionberger, R. A., and W. B. Russel, "High frequency modulus of hard sphere colloids," J. Rheol. 38(6), 1885-1908 (1994).

López-Barrón, C. R., L. Porcar, A. P. R. Eberle, and N. J. Wagner, "Dynamics of melting and recrystallization in a polymeric micellar crystal subjected to large amplitude oscillatory shear flow," Phys. Rev. Lett. 108(25), 258301 (2012).

Mackay, M. E., and B. Kaffashi, "Stress jumps of charged colloidal suspensions, measurement of the elasticlike and viscous-like stress components,” J. Colloid Interface Sci. 174, 117-123 (1995).

MacKintosh, F. C., and C. F. Schmidt, "Microrheology," Curr. Opin. Colloid Interf. Sci. 4, 300-307 (1999).

Maranzano, B. J., and N. J. Wagner, "Flow-small angle neutron scattering measurements of colloidal dispersion microstructure evolution through the shear thickening transition,” J. Chem. Phys. 117(22), 10291-10302 (2002).

Mason, T. G., and D. A. Weitz, "Optical measurements of frequency-dependent linear viscoelastic moduli of complex fluids,” Phys. Rev. Lett. 74, 1250-1253 (1995).

Mason T. G., K. Ganesan, J. H. van Zanten, D. Wirtz, and S. C. Kuo, "Particle tracking microrheology of complex fluids," Phys. Rev. Lett. 79(17) 3282-3285 (1997).

Meyer, A., A. Marshall, B. G. Bush, and E. M. Furst, "Laser tweezer microrheology of a colloidal suspension," J. Rheol. 50, 77-92 (2006).

Morris, J. F., and J. F. Brady, "Self diffusion in sheared suspensions," J. Fluid. Mech. 312, 223-252 (1996).

Perrin, J-.B., "Mouvement Brownien et réalité moléculaire (Brownian movement and the molecular reality)," Ann. Chim. Phys. 18, 5-114 (1909).

Pipkin, A. C., Lectures on Viscoelasticity Theory (Springer-Verlag, New York, 1986).

Rogers, S. A., J. Kohlbrecher, and M. P. Letting, "The molecular origin of stress generation in worm-like micelles, using a rheo-sans LAOS approach,” Soft Matter 8, 7831-7839 (2012).

Rogers, S. A., and M. P. Lettinga, "A sequence of physical processes determined and quantified in largeamplitude oscillatory shear (laos): Application to theoretical nonlinear models," J. Rheol. 56, 1-25 (2012).

Russel, W. B., D. A. Saville, and W. R. Schowalter, Colloidal Dispersions (Cambridge University Press, Cambridge, 1989).

Schultz, K. M., and E. M. Furst, "High-throughput rheology in a microfluidic device," Lab Chip 11, 3802-3809 (2011).

Squires, T. M., and J. F. Brady, “A simple paradigm for active and nonlinear microrheology,” Phys. Fluids 17, 073101 (2005).

Swan, J. W., and R. N. Zia, “Active microrheology-Fixed-velocity versus fixed-force,” Phys. Fluids 25, 083303 (2013).

Wagner, N. J., and J. F. Brady, "Shear thickening in colloidal dispersions," Phys. Today 62(10), 27-32 (2009). 
Watanabe, H., M.-L. Yao, K. Osaki, T. Shikata, H. Niwa, and Y. Morishima, "Nonlinear rheological behavior of a concentrated spherical silica suspension," Rheol. Acta 35(5), 433-445 (1996b).

Watanabe, H., T. Sato, and K. Osaki, "Relaxation of spherical micellar systems of styrene-isoprene diblock copolymers. 2. Nonlinear stress relaxation behavior," Macromolecules 29, 3890-3897 (1996a).

Wilhelm, W., "Fourier-transform rheology,” Macromol. Mater. Eng. 287, 83-105 (2002).

Wilson, L. G., A. W. Harrison, A. B. Schofield, J. Arlt, and W. C. K. Poon, "Passive and active microrheology of hard-sphere colloids," J. Phys. Chem. B 113, 3806-3812 (2009).

Wüstneck, R., B. Moser, and U. Pison, "Surface dilatational behavior of pulmonary surfactant components spread on the surface of a pendant drop. 2. Dipalmitoyl phosphatidylcholine and surfactant protein B," Langmuir 18, 1125-1130 (2002).

Zia, R. N., and J. F. Brady, "Single particle motion in colloids: Force-induced diffusion," J. Fluid Mech. 658, 188-210 (2010).

Zia, R. N., and J. F. Brady, "Microviscosity, microdiffusivity, and normal stresses in colloidal dispersions," J. Rheol. 56(5), 1175-1208 (2012).

Zia, R. N., and J. F. Brady, "Stress development, relaxation, and memory in colloidal dispersions: Transient nonlinear microrheology," J. Rheol. 57(2), 457-492 (2013).

Ziemann, F., J. Radler, and E. Sackmann, "Local measurements of viscoelastic moduli of entangled actin networks using an oscillating magnetic bead micro-rheometer," Biophys. J. 66, 2210-2216 (1994).

See supplementary material at http://dx.doi.org/10.1122/1.4826939 for the video that reflects the conditions $\mathrm{Pe}=3, \alpha=0.3$ and $\mathrm{Pe}=30, \alpha=3$. 